als nur ein symbolischer Anspruch auf dem (Verfassungs-)Papier, ob es in der praktischen Anwendung bestehen und von den demokratischen Produktivkräften in der Gesellschaft genutzt werden kann - dafür spielt die Dauer der Eintragungsfrist neben der Quorengestaltung im Gesamtgefüge der Volksgesetzgebung eine wichtige Rolle. Und deshalb bedarf es gewisser Mindeststandards für diese Frist.

\title{
Wechselfälle des Wahlrechts in Hamburg. Oder: Die Verhinderung eines weiteren Volksentscheids
}

\author{
Klaus David
}

Bis 2004 galt in Hamburg ein reines Verhältniswahlrecht mit gebundenen Listen, das 1956 eingeführt worden war. ${ }^{1}$ Es galt für die Wahlen sowohl zur Bürgerschaft als auch zu den Bezirksversammlungen. Die Parteien stellten die Kandidaten auf. Jeder Wahlberechtigte hatte eine Stimme für die Liste seiner Wahl. Dieses System bildete die Grundlage für 15 Wahlen bis zu dem erfolgreichen Volksentscheid am 13. Juni 2004 über ein neues Recht der Wahl zur Hamburgischen Bürgerschaft und zu den Bezirksversammlungen ${ }^{2}$, der zu einem grundlegenden Systemwechsel führte.

In den folgenden fünf Jahren spielten sich immer neue Kontroversen über das hamburgische Wahlrecht zwischen dem Volksgesetzgeber und der Initiative „Mehr Demokratie Ein faires Wahlrecht für Hamburg" einerseits und dem parlamentarischen Gesetzgeber andererseits sowohl über den Inhalt des Wahlrechts als auch über die wechselseitige Verbindlichkeit der jeweiligen Entscheidungen ab. ${ }^{3}$ 2009, als ein weiterer, aussichtsreich erscheinender Volksentscheid ${ }^{4}$ anstand, kam es schließlich zu einem ausgehandelten Kompromiss. Der Volksentscheid sollte am 27. September 2009, am Tag der Wahl zum Deutschen Bundestag, stattfinden. Im Sommer verständigten sich die Bürgerschaft und die Volksinitiatoren auf ein Wahlrecht einschließlich einer damit verbundenen Verfassungsänderung. ${ }^{5}$ Dieses Wahlrecht unterscheidet sich nicht wesentlich von dem des Jahres 2004;

1 Vgl. jeweils $\$ 4$ Abs. 1 der Gesetze über die Wahl zur hamburgischen Bürgerschaft vom 6 . Dezember 1956, GVBl., S. 497, S. 513 und in der Fassung der Bekanntmachung vom 22. Juli 1986, HmbGVBl., S. 223 mit nachfolgenden Änderungen.

2 Vgl. Gesetz zur Änderung des Gesetzes über die Wahl zur hamburgischen Bürgerschaft, des Gesetzes über die Wahl zu den Bezirksversammlungen, des Bezirksverwaltungsgesetzes und des Hamburgischen Meldegesetzes vom 5. Juli 2004, HmbGVBl., S. 313; in Kraft getreten am 15. Juli 2004.

3 Rechtspolitische und parteitaktische Erwägungen dieser Kontroverse hat Frank Decker dargestellt; ders., Parlamentarische Demokratie versus Volksgesetzgebung, Der Streit um ein neues Wahlrecht in Hamburg, in: ZParl, 38. Jg. (2007), H. 1, S. 118 - 133.

4 Vgl. die Mitteilungen des Senats an die Bürgerschaft - Bürgerschafts-Drs. 19/123 vom 8. April 2008, „Feststellung des Senats über das Zustandekommen einer Volksinitiative“ (15.327 Unterschriften) und 19/2534 vom 10. März 2009, „Feststellung des Senats über das Zustandekommen eines Volksbegehrens“, (76.086 Unterschriften).

5 Vgl. Zwölftes Gesetz zur Änderung der Verfassung der Freien und Hansestadt Hamburg vom 8. Juli 2009, HmbGVBl., S. 221. 


\begin{tabular}{|c|c|c|c|c|c|c|c|c|c|c|c|c|}
\hline & 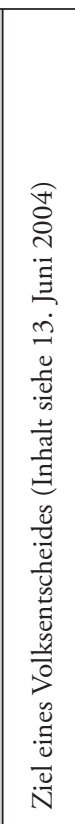 & 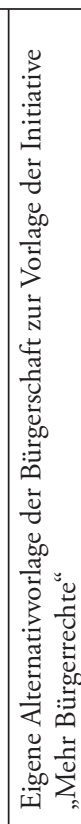 & 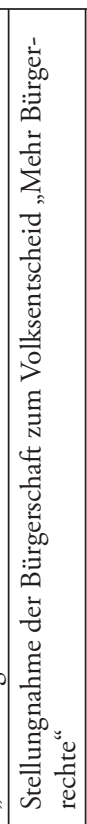 & 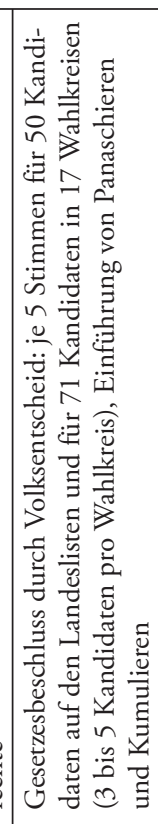 & 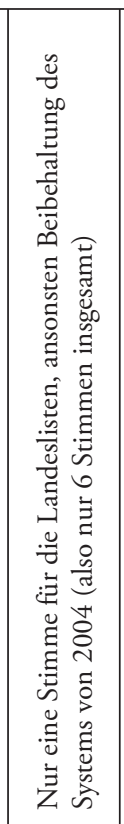 & 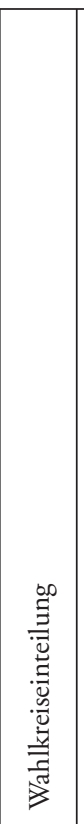 & 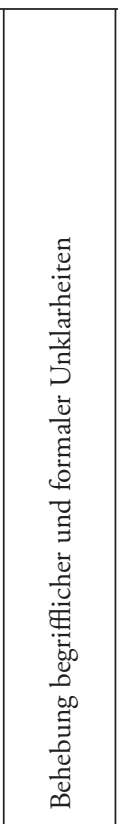 & 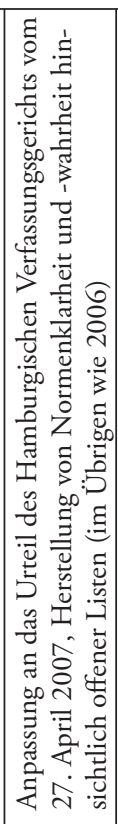 & 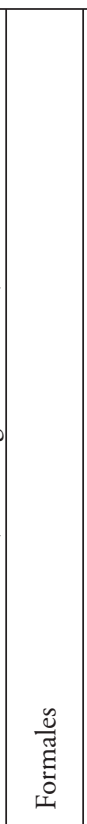 & 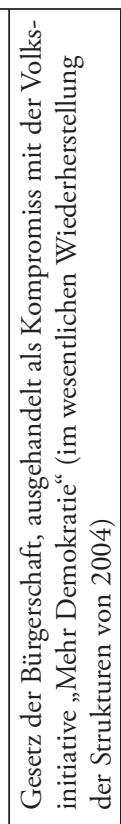 & 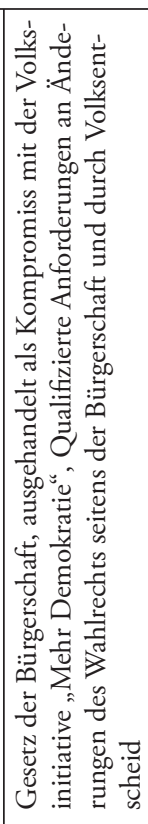 & \\
\hline 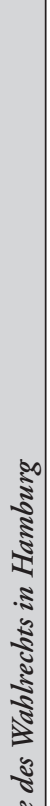 & 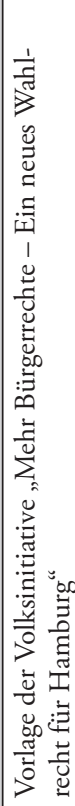 & 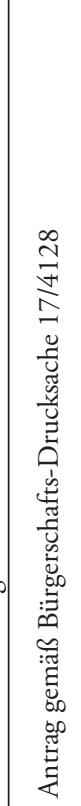 & 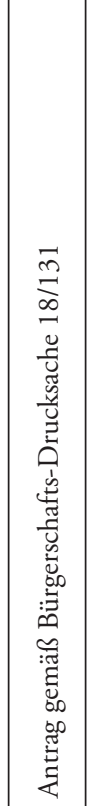 & 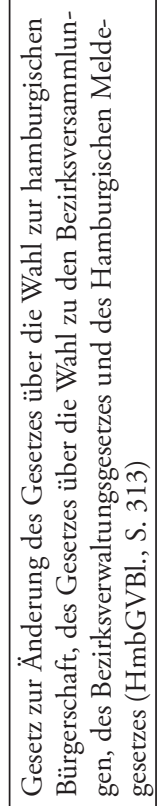 & 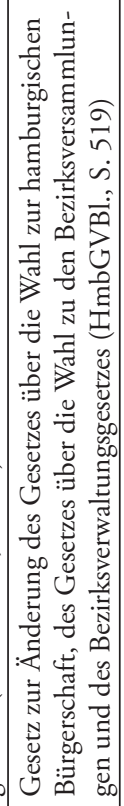 & 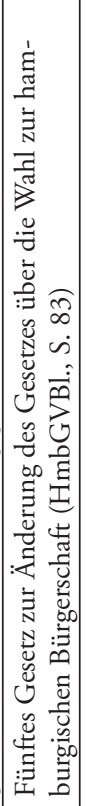 & 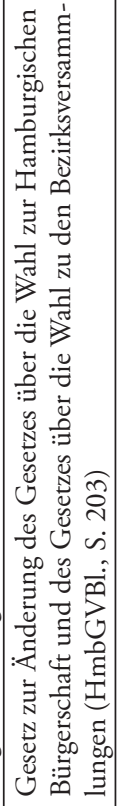 & 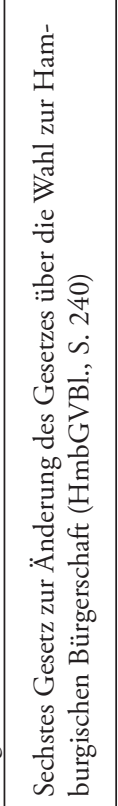 & 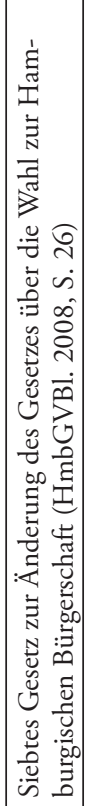 & 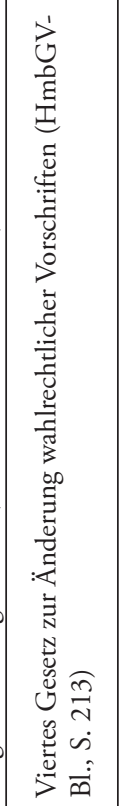 & 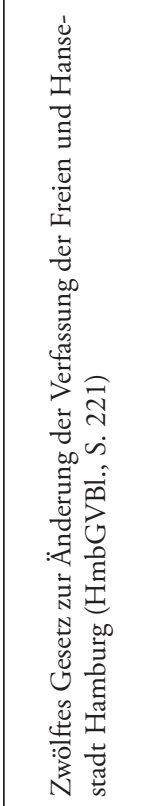 & 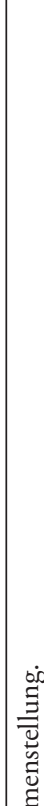 \\
\hline 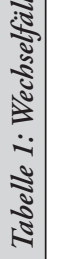 & 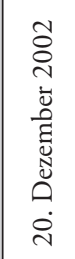 & 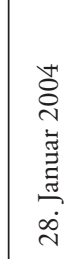 & 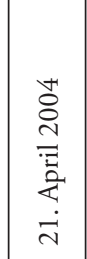 & 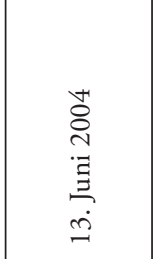 & 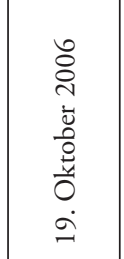 & 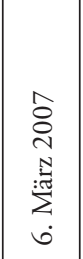 & 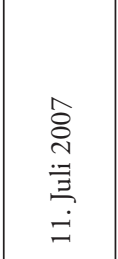 & $=$ & 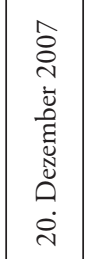 & $\begin{array}{l}\text { oे } \\
\text { ㄱ. } \\
\stackrel{y}{\exists}\end{array}$ & $\infty$ & \\
\hline
\end{tabular}


die Einigung wurde vielmehr maßgeblich von dem politischen Motiv diktiert, „die Auseinandersetzungen der vergangenen Jahre endlich beenden zu können“, und weiter „entscheidend war, dass die Volksparteien den Volksentscheid vermeiden wollten "6. Welche Wechselfälle des Wahlrechts diese fünf Jahre den Hamburgern bescherten, wird aus Tabelle 1 deutlich.

\section{Das Wahlrecht des Volksentscheids von 2004}

Die Volksinitiative „Mehr Bürgerrechte - Ein neues Wahlrecht für Hamburg“ begründete den 2004 vorgelegten Gesetzentwurf für ein neues Wahlrecht wie folgt: „Mit dem vorgeschlagenen Gesetz soll Hamburg ein neues Wahlrecht erhalten, damit die Wähler - aber auch die einzelnen Mitglieder der Parteien - mehr Einfluss auf die personelle Zusammensetzung der Bürgerschaft und der Bezirksversammlungen nehmen können. " schlag, eingereicht am 20. Dezember 2002, griff die Kritik auf, die bereits die EnqueteKommission „Parlamentsreform“ 1992 geäußert hatte. ${ }^{8}$ Die Initiatoren beklagten, dass die Parteien trotz der damaligen Kommissionsempfehlungen fast nichts unternommen hätten, um das Wahlrecht zu reformieren. „Deshalb ist es notwendig, über ein neues Wahlrecht die Bürgerinnen und Bürger selbst entscheiden zu lassen." Vorgeschlagen wurde ein stark personalisiertes Verhältniswahlrecht mit dem Ziel, den Einfluss der Wähler zu stärken und sinkenden Wahlbeteiligungen entgegenzuwirken. Nach dem Willen der Initiatoren sollte dies insbesondere durch die Einführung von Mehrpersonenwahlkreisen mit drei bis fünf Mandaten erreicht werden. Sowohl auf der Landesliste als auch in den Wahlkreisen sollte panaschiert und kumuliert werden können.

Die Bürgerschaft hatte daraufhin zu Ende ihrer 17. Wahlperiode 2004 von der ihr in Art. 50 Abs. 3 Satz 3, heute Satz 6 HV eingeräumten Möglichkeit Gebrauch gemacht, dem aus dem Volksbegehren hervorgegangenen Gesetzentwurf einen eigenen beizufügen und in der Volksabstimmung zur Entscheidung zu stellen. Dieser sah 50 Wahlkreise mit je circa 25.000 Wahlberechtigten vor. Bei 50 über Einmandatswahlkreise und 71 über Landeslisten zu erwerbenden Sitzen hätte sich das Zahlenverhältnis von 50 zu 71 im Vergleich zum Volkswahlgesetz umgekehrt. Mit zwei Stimmen, einer Wahlkreisstimme und einer für die Landesliste, war Panaschieren und Kumulieren ausgeschlossen.

\subsection{Déjà-vu? Nein}

Bereits 1949 sah das Gesetz über die Wahl zur hamburgischen Bürgerschaft ${ }^{9}$ (BüWG) ein durch Verhältnisausgleich abgewandeltes Mehrheitswahlrecht vor. Drei Fünftel der Abge-

6 Hamburger Abendblatt vom 12. Mai 2009, 25. Mai 2009, 30. Mai bis 1. Juni 2009 und 11. Juni 2009.

7 Vgl. Bekanntmachung über den Volksentscheid „Mehr Demokratie - Ein neues Wahlrecht für Hamburg", in: Amtlicher Anzeiger, Nr. 53 vom 7. Mai 2004, S. 889 ff., S. 894.

8 Vgl. Bürgerschafts-Drs. 14/2600 vom 20. Oktober 1992; Wolfgang Hoffmann-Riem (Hrsg.), Bericht der Enquete-Kommission „Parlamentsreform“, Baden-Baden 1993.

9 Vgl. GVBl. vom 19. August 1949, S. 169. 
ordneten wurden danach in Wahlkreisen, zwei Fünftel nach dem Grundsatz der Verhältniswahl nach Landeslisten gewählt. Das BüWG in der Fassung von $1952^{10}$ sah 72 Wahlkreise, also 72 Direktmandate, und 48 von damals 120 Abgeordneten vor, die nach den Grundsätzen der Verhältniswahl gewählt wurden.

Auf den ersten Blick wies das von 1949 bis 1956 geltende System hinsichtlich der Proportionen zwischen der Zahl der Direktmandate und der Listenmandate verblüffende Ähnlichkeit mit dem von der Volksinitiative vorgeschlagenen und 2004 beschlossenen Wahlrecht auf. Der zweite Blick zeigt: Partizipatorische Vorstellungen zur Stärkung des Wählereinflusses lagen dem Wahlsystem von 1949 keineswegs zugrunde. Das von 1919 bis 1933 geltende Verhältniswahlrecht sollte nicht wieder eingeführt werden, „da die durch dieses Wahlrecht begünstigte Zersplitterung des politischen Lebens mit zur Untergrabung der Weimarer Republik geführt hat"11. Der Gesetzgeber versprach sich also 1949 vom neuen System eine Wirkung, die der Fünfprozenthürde zugeschrieben wird, nämlich eine Stärkung der Handlungsfähigkeit des Parlaments statt seiner Zersplitterung. Dem trug das Gesetz 1956 bei der Rückkehr zum reinen Verhältniswahlrecht mit der Einführung der Fünfprozentklausel Rechnung. ${ }^{12}$ Der Rückkehr lag ein Kompromiss zugrunde. Die SPD strebte ein Verbot von Wahlvorschlägen durch Parteiverbindungen an, die sie in dem von 1953 bis 1957 regierenden so genannten Hamburg-Block gesehen hatte. Sie stimmte im Gegenzug der Aufhebung der Wahlkreise zu, deren Beibehaltung sie ursprünglich befürwortet hatte. ${ }^{13}$

\subsection{Die Grundsätze des Volkswahlgesetzes}

Im Volksentscheid am 13. Juni 2004 wurde der Entwurf der Bürgerschaft abgelehnt ${ }^{14}$, die Vorlage der Volksinitiative angenommen. Das so beschlossene Gesetz stellte ein Artikelgesetz zur Änderung von vier geltenden Gesetzen dar, nicht dagegen ein in sich geschlossenes vollständig neues Gesetz. Damit wurde das Wahlrecht wie folgt geändert ${ }^{15}$ :

(1) In 17 Wahlkreisen sollten mindestens 71 der 121 Abgeordneten nach offenen Wahlkreislisten in Mehrmandatswahlkreisen gewählt werden. Abhängig von der Zahl der dort jeweils Wahlberechtigten konnten drei, vier oder fünf Kandidaten gewählt werden. Einzelbewerber waren zugelassen. 50 Sitze waren nach offenen Landeslisten für Hamburg insgesamt zu besetzen. Eine Fünfprozenthürde sollte gelten.

10 In der Fassung vom 29. November 1952, GVBl. S. 257.

11 Wilhelm Drexelius / Renatus Weber, Die Verfassung der Freien und Hansestadt Hamburg - Kommentar, Hamburg 1953, Art. 6, S. 8, Anmerkung 5.

12 Vgl. $\$ 5$ Abs. 2 des Gesetzes von 1956, a.a.O. (Fn. 1); $\$ 5$ Abs. 2 des Gesetzes von 1986, a.a.O. (Fn. 1).

13 Vgl. Stenographischer Bericht über die 23. Sitzung der Bürgerschaft am 28. November 1956, S. 915, S. 929 ff.; Hans Peter Ipsen, Hamburgs Verfassung und Verwaltung, Hamburg 1956, Aalen 1988, S. 488.

14 Vgl. Bekanntmachung des Ergebnisses des Volksentscheids „Mehr Bürgerrechte - Ein neues Wahlrecht für Hamburg“, in: Amtlicher Anzeiger, Nr. 77 vom 5. Juli 2004, S. 1343. Der Senat machte nur den Erfolg des Volksentscheids bekannt, nicht aber den Misserfolg der Vorlage der Bürgerschaft. Er ergibt sich nur mittelbar.

15 Vgl. die Darstellung der Grundzüge bei Günter Hoog, Hamburgs Verfassung, Baden-Baden 2004, S. $81 \mathrm{f}$. 
(2) Zweimal fünf Stimmen, je fünf für die Wahlkreis- und die Landeslisten, sollten beliebig auf die Wahlvorschläge und die in ihnen genannten Personen verteilt werden. Kumulieren und Panaschieren war also zugelassen.

(3) Die Verteilung der mindestens 121 Sitze richtete sich nach dem Verhältnis der Parteistimmen für die Wahl nach den Landeslisten. Die über die Wahlkreislisten zu vergebenden Sitze wurden nach denselben Grundsätzen zugeteilt, die für die Verteilung nach den Landeslisten gelten sollten.

(4) Die Gesamtzahl der Mandate hätte sich nicht nur um die Überhangmandate und Ausgleichsmandate erhöht, die vergeben werden sollten, um die Sitzverteilung insgesamt nach dem Verhältnis der Parteistimmen zu gewährleisten. Dadurch und durch erfolgreiche Einzelbewerber konnte sich die Gesamtzahl der Sitze in der Bürgerschaft auf über 121 erhöhen, abhängig vom jeweiligen Wahlergebnis.

(5) Die Wahlen zu den Bezirksversammlungen sollten entsprechend dem Recht der Wahlen zur Bürgerschaft durchgeführt werden, soweit nicht etwas anderes in diesem Gesetz bestimmt war.

Diese Neuregelungen sollten erstmalig auf die Wahlen zur Bürgerschaft und zu den Bezirksversammlungen am 24. Februar 2008 angewendet werden. Dazu kam es wegen nachfolgender parlamentarisch beschlossener Änderungen aber nicht.

\section{Das Wahlgesetz von 2006}

Im Juni 2006 brachte die CDU-Fraktion einen Antrag zur Änderung des Wahlgesetzes in die Bürgerschaft ein. ${ }^{16}$ Am 27. September 2006 wurde dieser in erster Lesung mehrheitlich ${ }^{17}$ und am 11. Oktober in zweiter Lesung mit 62 der 63 Stimmen der Fraktion der CDU gegen die Stimmen der Fraktionen von SPD und GAL (Grün-Alternative Liste) ${ }^{18}$ angenommen. Das Gesetz, das die geltende Rechtslage erheblich änderte, trat am 28. Oktober 2006 in Kraft.

\subsection{Ziel der Gesetzesänderung}

Die CDU-Fraktion nannte zwei Ziele, denen die Änderung des Volkswahlgesetzes dienen sollte: primär die Sicherung der Funktions- und Handlungsfähigkeit des Parlaments ${ }^{19}$ und sodann die Vereinfachung des Wahlvorgangs. Das erste Ziel sei durch das Volkswahlrecht gefährdet, weil es „den Parteien jede Einflussmöglichkeit auf die Reihenfolge der Landesliste nahm. Denn neben der Sitzverteilung zwischen den Parteien und Wählergemeinschaften ist auch die personelle Zusammensetzung der Bürgerschaft von entscheidender Bedeutung "20. Auf den vorderen Listenplätzen sollten nach Meinung der Antragsteller

16 Vgl. Bürgerschafts-Drs. 18/4339 (Neufassung) vom 19. Juni 2006.

17 Vgl. Plenarprotokoll 18/64 vom 27. September 2006 über die Sitzung am 27. September 2006, S. 3358B - 3378B, S. $3404-3405$.

18 Vgl. Plenarprotokoll 18/65 vom 11. Oktober 2006 über die Sitzung am 11. Oktober 2006, S. 3407, S. 3459B; Frank Decker, a.a.O. (Fn. 3), S. 123, S. 130 f.

19 Vgl. Bericht des Verfassungsausschusses, Bürgerschafts-Drs. 18/4889 vom 28. August 2006, S. 8.

20 Vgl. Antrag, Bürgerschafts-Drs. 18/4339, a.a.O. (Fn. 16), S. 6 zu Nr. 2 (\$ 3), Ziffer 2.3. 
Kandidaten abgesichert werden können, die sich innerparteilich oder im Rahmen der parlamentarischen Arbeit als Experten für bestimmte Fachthemen qualifiziert hätten. Der Einfluss von Parteien auf die personelle Zusammensetzung des Parlaments gehöre zu den zentralen Elementen einer Parteiendemokratie.

\subsection{Die Grundsätze der Novelle von 2006}

(1) Die Novelle behielt zwar die 17 Mehrmandatswahlkreise bei, in denen insgesamt 71 der 121 Abgeordneten zu wählen waren, je nach Größe wie nach dem Volkswahlgesetz drei, vier oder fünf Kandidaten. Auch Einzelbewerber waren zugelassen.

(2) Die Wahlberechtigten sollten aber nur sechs Stimmen haben, davon fünf für die Wahl nach Wahlkreislisten. Sie sollten wie nach dem Volkswahlgesetz von 2004 durch Kumulieren und Panaschieren auf die Wahlkreiskandidaten oder auf die Wahlkreislisten in ihrer Gesamtheit gesetzt werden können. Bei nur einer Stimme für die Landesliste war dies dafür ausgeschlossen. Einzelbewerber waren zugelassen. Es galt die Fünfprozenthürde.

(3) Die errungenen Direktmandate sollten in der Reihenfolge der erhaltenen Persönlichkeitsstimmen nur solchen Kandidaten zugeteilt werden, die die so genannte Relevanzschwelle übersprungen hatten. ${ }^{21}$ Die weiteren einem Wahlvorschlag zugefallenen Sitze wurden den Personen in der Reihenfolge der Wahlkreisliste zugewiesen. Das Gesetz hatte damit die Möglichkeiten des Kumulierens und Panaschierens gemäß dem Volkswahlgesetz von 2004 erheblich erschwert. Im Ergebnis handelte es sich nun für die Wahlkreise um eine Verhältniswahl nach nur eingeschränkt offenen Listen.

(4) Daran schloss sich die Vergabe der restlichen 50 Sitze in der Reihenfolge ihrer Nennung auf der Landesliste an. Hinzu kamen unter anderem Überhang- und Ausgleichsmandate und schließlich Mandate gemäß einer so genannten Mehrheitssicherungsklausel. Mit ihr sollte sichergestellt werden, dass eine Partei oder Wählervereinigung, die gemäß dem Ergebnis der Landeslistenwahl die absolute Mehrheit errungen hatte, die absolute Mehrheit der Bürgerschaftsmandate auch in dem Fall behielt, wenn sie ihr nach dem genannten Verfahren verloren gegangen war. Ihr wurden dann so viele Sitze zusätzlich zugeteilt, wie nach Ermittlung der Gesamtzahl der Sitze in der Bürgerschaft dazu erforderlich waren. Damit wurde ein Element der Mehrheitswahl in das Verhältniswahlrecht eingefügt. Die Wirkung des Persönlichkeitselements wurde über die Relevanzschwelle hinaus weiter reduziert. $^{22}$

Die maßgebende Rolle, die die Parteien und Wählervereinigungen damit erhalten sollten, rechtfertigte die CDU-Fraktion mit der verfassungsrechtlichen Stellung der Parteien gemäß Art. 21 Abs. 1 Satz 1 GG. Nach ihrer Auffassung sei ihnen damit über die Mitwirkung bei der Willensbildung des Volkes hinaus zugewiesen, bei der Vorformung und Organisation des politischen Willens, bei der Kreation der Staatsorgane sowie bei der inhaltlichen Ausrichtung der politischen Entscheidungen eine wesentliche Funktion zu erfüllen. ${ }^{23}$

In der Fassung vom Oktober 2006 konnte das Wahlrecht indes nicht Grundlage der

21 Einzelheiten sind entbehrlich, da die Relevanzschwelle 2007 abgeschafft wurde.

22 Vgl. Hans Peter Bull, Stellungnahme, Protokoll über die Sitzung des Verfassungsausschusses am 21. Juni 2006 - APr. 18/22, S. 63, S. 65; Anhörung am 21. Juni 2006, S. 27.

23 Vgl. Bericht des Verfassungsausschusses, a.a.O. (Fn. 19), S. 9. 
Wahlen vom 24. Februar 2008 werden. Es sah sich zwei Verfahren vor dem Hamburgischen Verfassungsgericht ausgesetzt. Anschließend novellierte es der Gesetzgeber einmal mehr.

\section{Verfahren vor dem Hamburgischen Verfassungsgericht}

Das erste Verfahren hatten die Organisatoren der Volksinitiative „Mehr Bürgerrechte - Ein neues Wahlrecht für Hamburg" angestrengt. Gegenstand war die Frage, ob die Bürgerschaft das Recht hatte, das Wahlrechtsänderungsgesetz vom 19. Oktober 2006, zu beschließen, mit dem sie das im Wege eines Volksentscheids beschlossene Wahlrecht teilweise geändert hatte. Sie hatten einen Antrag gestellt mit dem Ziel, das Verfassungsgericht möge feststellen, die Bürgerschaft habe mit dem Gesetzesbeschluss die ihr nach dem Grundsatz der Organtreue verfassungsrechtlich obliegende Treuepflicht gegenüber den Hamburger Bürgern und ihr selbst als Verfassungsorgan verletzt. Das habe die Nichtigkeit des gesamten Wahlrechtsänderungsgesetzes zur Folge. Das Verfassungsgericht sprach aber der Antragstellerin die Parteifähigkeit ab, weil sie weder ein Verfassungsorgan noch eine andere Beteiligte sei, wobei zwei Richter dieser Auffassung in einer „Abweichenden Meinung“widersprachen.

In einem zweiten Verfahren hatten 58 Mitglieder der Bürgerschaft aus den Fraktionen der SPD und der GAL einen Normenkontrollantrag nach Art. 65 Abs. 3 Nr. 3 HV, $\$ \$ 14$ Nr. 3, 40 ff. Gesetz über das Hamburgische Verfassungsgericht (HVerfGG) gestellt mit dem Ziel der Feststellung, dass das Wahlrechtsänderungsgesetz die Verfassung verletze und nichtig sei, weil es das im Wege eines Volksentscheides beschlossene Wahlrecht teilweise änderte. Außerdem bestritten sie die Verfassungsmäßigkeit von vier Bestimmungen des Änderungsgesetzes. Von den vier im Einzelnen gestellten Anträgen gab das Verfassungsgericht in seinem Urteil vom 27. April $2007^{24}$ nur einem statt, erklärte im Übrigen aber das Änderungsgesetz für vereinbar mit der Verfassung.

\subsection{Organtreue}

Das Verfassungsgericht stellte fest, dass zwischen dem Volks- und dem Parlamentsgesetzgeber das Prinzip der Organtreue gelte. ${ }^{25}$ Dieser für das Grundgesetz entwickelte ungeschriebene Rechtsgrundsatz ${ }^{26}$ sei ebenfalls für die Verfassungen der Länder anzuwenden. ${ }^{27}$ Des Weiteren befanden die Richter, dass die Bürgerschaft mit dem Erlass des Änderungsgesetzes die aus dem Grundsatz der Organtreue gegenüber dem Volksgesetzgeber folgenden Pflich-

24 Vgl. HVerfG 04/06 - HmbJVBl. 2007, S. 60, S. 68, S. 77; bekannt gemacht am 3. Mai 2007, HmbGVBl., S. 159 .

25 Vgl. HVerfG 04/06 - HmbJVBl. 2007, a.a.O. (Fn. 24), S. 72 f; HVerfG, Urteil vom 15. Dezember 2004 - HVerfG 6/04, HmbJVBl. 2005, S. 19, S. 29 f.; DÖV 2005, S. 252, S. 255; DVB1 2005, S. 438, S. 443 mit Anmerkung Klaas Engelken, S. 415 ff.; NVwZ 2005, S. 685, S. 688; NordÖR 2005, S. 109, S. 114 mit Anmerkung Hans Peter Bull, S. 99.

26 Vgl. BVerfG, Beschluss vom 4. Juni 1973, in: BVerfGE 35, S. 193, S. 199; Urteil vom 25. Mai 1977, in: BVerfGE 45, S. 1, S. 39.

27 Vgl. Klaus David, Kommentar zur Verfassung der Freien und Hansestadt Hamburg, Stuttgart 2004, Art. 50, Rn. 11; Martin Borowski, Parlamentsgesetzliche Änderungen volksbeschlossener Gesetze, in: DÖV 2000, S. 481, S. 490; HVerfG 04/06 - HmbJVBl. 2007, a.a.O. (Fn. 24), S. 72 f. 
ten nicht verletzt hat. ${ }^{28}$ Dabei gingen sie zunächst von der Gleichrangigkeit von Volks- und Parlamentsgesetzen aus. Mangels Vorrangs etwa eines Volksgesetzes vor einem Parlamentsgesetz lasse sich nicht im Wege der Organtreue eine Beschränkung der Gesetzgebungskompetenz der Bürgerschaft begründen. Daran ändere auch die Tatsache nichts, dass es sich im vorliegenden Fall um ein Wahlgesetz handele.

Das Gericht hat hingegen nicht festgestellt, dass die Bürgerschaft gegen die ihr obliegenden Prüfungs- und Berücksichtigungspflichten verstoßen habe. Die parlamentarischen Diskussions- und Abstimmungsprozesse seien durchgeführt, die Erkenntnis- und Beratungsmöglichkeiten in den parlamentarischen Fachgremien ausgeschöpft worden. Die Protokolle der Sitzungen des Verfassungsausschusses und dessen Bericht an das Plenum der Bürgerschaft zeigten dies. Zahlreiche Sachargumente seien angeführt worden, die im Urteil erörtert werden.

$\mathrm{Zu}$ seiner eigenen Position legt das Gericht dar, dass es sich einer eigenen Abwägung über den in Rede stehenden Gegenstand der politischen Willensbildung zu enthalten habe. Diese Zurückhaltung entspreche ihrerseits dem Gedanken der Organtreue. Die gerichtliche Überprüfbarkeit beschränke sich auf die Ermittlung, ob sich der Parlamentsgesetzgeber mit dem Volksgesetz überhaupt inhaltlich sachlich auseinandergesetzt habe. Das aber habe ihm das Verfassungsgericht bestätigt, so dass es weitere Erwägungen für den Fall eines negativen Ermittlungsergebnisses nicht habe anstellen müssen.

\subsection{Relevanzschwelle}

(1) Lediglich den Einzelantrag auf Feststellung, dass die Regelung über die Relevanzschwelle gegen den Grundsatz der Wahlgleichheit verstoße, erklärte das Verfassungsgericht für begründet - allerdings nicht aus diesem Grund. Vielmehr sei die mit der Relevanzschwelle verbundene Einschränkung der Möglichkeit, mittels Panaschierens und Kumulierens die Reihenfolge der Kandidaten der Wahlkreisliste zu beeinflussen, mit den in Art. 6 Abs. 2 HV verankerten Prinzipien der gleichen und der freien Wahl vereinbar. Die konkrete Regelung verletze aber die Grundsätze der Normenklarheit und -wahrheit.

(2) Die Antragsteller waren der Ansicht, die Relevanzschwelle verhindere die „Gleichheit im Stimmengewicht des Zählwerts in der Verhältniswahl“ hinsichtlich der Persönlichkeitsstimmen in Bezug auf die Personalauswahl innerhalb der Wahlkreisliste, da diese Stimmen zwar gezählt, aber nicht gewogen würden, es sei denn, sie erreichten die Relevanzschwelle. Diesen Stimmen komme also nicht derselbe Erfolgswert zu.

Das Verfassungsgericht hat diese Auffassung geteilt, aber die Differenzierung des Gleichheitsgrundsatzes nach Zähl- und Erfolgswert nicht für verfassungswidrig erklärt. Das Wahlsystem mit der Variante der Relevanzschwelle gebiete keine Erfolgswertgleichheit. Die Beschränkung der Gleichheit im Stimmengewicht auf Stimmen unterhalb der Relevanzschwelle stelle sich als Teil der zulässigen Ausgestaltung eines Verhältniswahlrechts mit nur eingeschränkt offenen Listen dar. ${ }^{29}$ Die Wahl der Kandidaten in den Wahlkreisen hat das Gericht als ein Wahlrecht nach starren Listen gekennzeichnet. Die Relevanzschwelle hat das Gericht lediglich als Gestaltungsvariante bezeichnet. Aus der Begründung des

28 Vgl. HVerfG 04/06 - HmbJVBl. 2007, a.a.O. (Fn. 24), S. 72 ff.

29 Vgl. ebenda, S. 79. 
Urteils ergibt sich nichts für die Annahme, dass in der rechtlichen Regelung der Relevanzschwelle vielleicht ein Element des Mehrheitswahlsystems liegen könnte, was die Geltung der Zählwertgleichheit hätte begründet erscheinen lassen. Für diese Annahme ließen sich Gründe finden, weil bei Überschreiten der Schwelle ein Kandidat sich mit der auf ihn entfallenden Stimmenzahl gegenüber zunächst vorrangig rangierenden Kandidaten durchsetzt. Wäre das Verfassungsgericht zu dieser Erkenntnis gelangt, hätte es das Wahlkreisrecht als Mischsystem von Verhältnis- und Mehrheitswahl kennzeichnen können. Mit der Bewertung der Relevanzschwelle als Element eines Mehrheitswahlrechts hätte das Gericht die Voraussetzung dafür geschaffen, den Grundsatz der Gleichheit der Wahl in Gestalt des gleichen Zählwerts für anwendbar und ausreichend zur Begründung der Verfassungsmäßigkeit des Wahlkreisrechts insgesamt vor Art. 6 Abs. 2 HV zu erklären.

Stattdessen hielt es aber an der prinzipiellen Einordnung des Systems als Verhältniswahl fest. Dann aber hätte durchgängig der Grundsatz der Erfolgswertgleichheit gelten müssen.

(3) Das Verfassungsgericht hat die gesetzliche Regelung der Relevanzschwelle allerdings deshalb verworfen, weil sie nicht den Anforderungen genüge, die gemäß den aus dem Rechtsstaatsprinzip nach Art. 3 Abs. 1 HV folgenden Geboten der Normenklarheit und Normenwahrheit zu stellen seien. Die Relevanzschwelle blieb damit als Institut verfassungsrechtlich intakt. Das Gebot der Normenklarheit erfordere, dass die von einer gesetzlichen Regelung Betroffenen die Rechtslage erkennen können und ihr Verhalten danach einzurichten vermöchten. Zur Normenklarheit gehöre auch die Normenwahrheit. Eine der Sachlage zuwiderlaufende Gesetzesgestaltung verstoße gegen das Rechtsstaatsgebot. Gemessen an diesen Maßstäben sei die in Art. $1 \$ 4$ Abs. 3 BüWG 2006 geregelte Relevanzschwelle nicht hinreichend klar gefasst. Dem Wähler, der seine Stimme für eine Person abgebe, werde ein falsches Bild vermittelt, weil er nicht erkennen könne, dass die ihm vor allem in Gestalt des Kumulierens und Panaschierens angebotenen Handlungsmöglichkeiten in Wirklichkeit eher theoretischer Natur seien und sich sogar kontraproduktiv auswirken könnten. $\$ 2$ Abs. 2 BüWG spreche irreführend von „offenen“ Wahlkreislisten. Das Wahlgesetz von 2006 hatte diesen Begriff sprachlich unverändert aus dem Volkswahlgesetz von 2004 übernommen, das aber die Relevanzschwelle nicht kannte. Allein für die Landeslisten ersetzte das Gesetz „offen“ durch „gebunden“. Die Wähler mussten davon ausgehen, dass in Anbetracht dessen der Begriff „offene Wahlkreislisten“ mit derselben Bedeutung erhalten blieb, die er 2004 besaß.

Darüber hinaus rechne nach Auffassung des Verfassungsgerichts auch der Listenstimmenwähler üblicherweise nicht damit, dass seine Stimme für die Liste einer Partei oder Wählervereinigung nur hinsichtlich der Sitzzahl, nicht aber hinsichtlich der in der Liste aufgeführten Reihenfolge der Kandidaten gewertet würde. Vielmehr sollten die Plätze für Kandidaten, die die Relevanzschwelle überschritten hätten, allein in der Reihenfolge der erhaltenen Persönlichkeitsstimmen vergeben werden. Dass die Listenstimmen bei der Verteilung der auf die Listen entfallenden Sitze gewissermaßen unter den Tisch fielen oder wie Enthaltungen behandelt würden, sei bei der Ausübung des Wahlrechts nicht klar. ${ }^{30}$ Das Verfassungsgericht beanstandete diese begrenzte Wertung nicht als solche, sondern allein die Unklarheit des Gesetzes, aus dem sich diese Begrenzung nicht mit der erforderlichen Klarheit ergebe. 


\subsection{Mehrheitssicherungsklausel}

Die so genannte Mehrheitssicherungsklausel stieß nicht auf die Kritik der Richter. ${ }^{31}$ In einem Proportionalverfahren wie dem geltenden, entspreche es bei Erreichen der absoluten Mehrheit einer Partei oder Wählervereinigung, die Verteilung der Sitze entsprechend abzubilden und dafür gegebenenfalls auch erforderliche zusätzliche Sitze zu gewähren. Das Verfassungsgericht verwies in diesem Zusammenhang unter anderem auf den Ausgleich von Überhangmandaten. Das maßgeblich nach den Stimmen für die Landeslisten zu bestimmende Sitzverteilungsverhältnis werde durch die Gewährung von Ausgleichsmandaten nicht berührt.

\subsection{Wahlen zu den Bezirksversammlungen: Fünfprozentklausel}

Das Verfassungsgericht hat seine Rechtsprechung zur Verfassungsmäßigkeit der Fünfprozentklausel für Wahlen zu den Bezirksversammlungen fortgesetzt. ${ }^{32}$

\section{Die Wahlgesetze von 2007}

Im Anschluss an das Urteil des Verfassungsgerichts brachte die Bürgerschaft vier Änderungen zum Bürgerschaftswahlgesetz, davon zwei mit Ausfertigungsdatum vom 11. Juli 2007, auf den Weg.

\subsection{Die beiden Änderungsgesetze vom 11. Juli 2007}

Das von diesen beiden zuerst publizierte, das Gesetz zur Änderung des Gesetzes über die Wahl zur hamburgischen Bürgerschaft und des Gesetzes über die Wahl zu den Bezirksversammlungen $^{33}$, da rf getrost als Maßnahme zur Behebung von begrifflichen und formalen Unklarheiten und Fehlern früherer Gesetze bezeichnet werden, nicht als eines zur materiellen Änderung des Wahlrechts - ein legislatorisches Unikat.

Das Verfassungsgericht hatte in seinem Urteil vom 27. April 2007 den Gesetzgeber gemahnt, für eine verfassungsgemäße Regelung zu sorgen, die der Normenklarheit hinsichtlich der Berücksichtigung der ausdrücklich vorgesehenen Listenstimmen bei der Sitzverteilung innerhalb der Wahlkreise genüge. In Anbetracht sowohl der bereits laufenden Vorbereitungen für die Wahl am 24. Februar 2008 als auch der sonst drohenden Wahlanfechtungen werde der Gesetzgeber diese Aufgabe schnellstmöglich zu erfüllen haben. ${ }^{34}$

31 Vgl. ebenda, S. $76 \mathrm{f}$.

32 Vgl. ebenda, S. 75 f. unter Verweis auf seine Urteile vom 6. November 1998 - HVerfG 1/98 -, HmbJVBl. 1999, S. 4, S. 6 und vom 26. November 1998 - HVerfG 4/98, HmbJVBl. 1999, S. 14, S. 23.

33 Vgl. HmbGVBl. 2007, S. 203.

34 Vgl. HVerfG 04/06 - HmbJVBl. 2007, a.a.O. (Fn. 24), S. 82. 
Diesem Monitum ist die Bürgerschaft mit dem Sechsten Gesetz zur Änderung des Gesetzes über die Wahl zur hamburgischen Bürgerschaft, ebenfalls vom 11. Juli 2007, nachgekommen. ${ }^{35}$ Das Wahlgesetz von 2006 in der Fassung der Änderungen von 2007 bildete schließlich die Grundlage für die Bürgerschaftswahl 2008.

\subsection{Die Strukturen des Wahlgesetzes von 2007}

(1) Prägende Merkmale blieben erhalten: die Einrichtung von 17 Mehrmandatswahlkreisen mit drei bis fünf zu wählenden Abgeordneten je Wahlkreis, insgesamt 71; die Gewährung von weiterhin sechs Stimmen, fünf für die Wahlkreisliste mit der Unterscheidung von Persönlichkeits- und Listenstimmen sowie die Möglichkeit des Panaschierens und Kumulierens nur in den Wahlkreisen. Einzelbewerber blieben zugelassen. Für die Landeslisten gab es nur eine Stimme. Die Relevanzschwelle entfiel. Die Fünfprozenthürde blieb.

(2) Dem Gebot der Normenklarheit und -wahrheit entsprechend verzichtete Art. $1 \$ 2$ Abs. 2 BüWG darauf, Listen als „offen“ zu bezeichnen. Der neu gefasste $\$ 4$ Abs. 3 ließ es auf Grund des darin vorgesehenen Verfahrens der wahlvorschlagsinternen Sitzverteilung zu, dass die auf einen Wahlvorschlag entfallenden Sitze allein nach der Listenreihenfolge vergeben werden. Deshalb gab es keine offenen Listen.

(3) Für die Sitzverteilung in den Wahlkreisen sah Art. $1 \$ 4$ Abs. 2 vor, dass zunächst errechnet wurde, wie viele Sitze auf jeden einzelnen Wahlvorschlag entfielen. Sodann wurde nach $\$ 4$ Abs. 3 bis 5 ermittelt, welche der in einem Wahlvorschlag benannten Personen die auf diesen entfallenden Sitze erhielten. Ausgangspunkt dieses Systems blieb die auf das Volkswahlgesetz von 2004 zurückzuführende Festlegung, dass der Wähler weiterhin die Möglichkeit haben sollte, seine bis zu fünf Wahlkreisstimmen als Persönlichkeitsstimmen für bestimmte Kandidaten, als Listenstimmen an Wahlkreislisten in ihrer Gesamtheit oder verteilt auf beide zu vergeben. Mit Listenstimmen favorisieren Wähler nicht nur die Partei oder Wählervereinigung, die die Liste aufgestellt hat, sondern auch die damit verbundene Reihenfolge der Sitzverteilung. Mit den Persönlichkeitsstimmen entscheiden die Wähler über die Sitzverteilung gemäß den von den Bewerbern jeweils erreichten Stimmenzahlen.

Der Forderung des Verfassungsgerichts, dass sich die Listenstimmen nicht nur auf die Zahl der zu vergebenden Sitze, sondern auch auf deren Verteilung auf die Bewerber auswirken müssten, trug der geänderte Art. $1 \$ 4$ Abs. 3 Satz 1 BüWG Rechnung. Die Sätze 2 und 3 bestimmten, dass zur Ermittlung der Sitzverteilung der Anteil der Listenstimmen und jener der Persönlichkeitsstimmen an den auf einen Wahlvorschlag insgesamt entfallenden Stimmen, den Parteistimmen, festgestellt wurde. Die konkrete Sitzverteilung vollzog sich dann nach der Höhe dieser Anteile. Waren Sitze sowohl nach der Personen- als auch nach der Wahlkreisliste zuzuteilen, wurden sie nach $\$ 4$ Abs. 3 Satz 1 ,anteilig in der Reihenfolge der Listenplatzierung und der Persönlichkeitsstimmenzahlen“ vergeben. Die weiteren Alternativen kennzeichnete Satz 1 mit ,allein in der Reihenfolge der Listenplatzierung oder der Persönlichkeitsstimmenzahl“. Diese Alternativen kamen zum Zuge, wenn auf einen Wahlvorschlag nur ein Sitz entfiel. Welche davon griff, hing von der Zahl der Stimmen ab, die eine Wahlkreisliste beziehungsweise ein Bewerber erzielte. Erhielt eine 
Liste die höhere Stimmenzahl, bekam der erstplatzierte Listenbewerber den Zuschlag, im umgekehrten Fall der Bewerber mit der höchsten Zahl an Persönlichkeitsstimmen. ${ }^{36}$ Die Zahl der von einer Partei oder Wählervereinigung in einem Wahlkreis errungenen Sitze wurde gemäß $₫ 5$ Abs. 5 von der für jede Landesliste ermittelten Zahl der Abgeordneten abgerechnet.

\subsection{Das Wahlprüfungsverfahren 2008/2009}

Auch das Bürgerschaftswahlgesetz in der Fassung von 2007 wurde einer verfassungsgerichtlichen Überprüfung unterzogen, und zwar in Gestalt eines Wahlprüfungsverfahrens. Die Bürgerschaft hatte den Wahleinspruch auf Empfehlung des Verfassungs- und Bezirksausschusses ${ }^{37}$ zurückgewiesen. ${ }^{38}$ Dagegen legte der Einsprechende Beschwerde beim Verfassungsgericht ein. Er vertrat die Auffassung, dass die in Art. $1 \$ 4$ BüWG in der Fassung von 2007 geregelte Verteilung der Sitze in den Wahlkreisen nach Persönlichkeits- und Listenstimmen, vor allem die sich aus den Absätzen 4 und 5 ergebende Wirkung der Reihenfolge der Zuteilung nach Art der Stimmen, den Grundsatz der Unmittelbarkeit der Wahl sowie das Gebot der Normenklarheit verletze. $\$ 4$ Abs. 3 bis 5 lasse nicht erkennen, dass der Wähler mit der Abgabe der Persönlichkeitsstimmen für einen Bewerber auf einem hinteren Platz der Wahlkreisliste nur sehr geringe Erfolgschancen habe, auch wenn er eine höhere Stimmenzahl auf sich vereinige als Bewerber auf vorderen Rängen. Diese zögen trotz geringerer Stimmenzahl in die Bürgerschaft ein. Der Wähler, der Bewerbern auf den hinteren Rängen seine Stimme gebe, verhelfe jenen auf den vorderen Plätzen zum Mandatserwerb. Das widerspreche dem Grundsatz der Unmittelbarkeit der Wahl und verletze das Gebot der Normenwahrheit und -klarheit.

Art. 6 Abs. 2 HV enthält den Grundsatz der Unmittelbarkeit der Wahl. Er fordert die direkte Wahl der Abgeordneten und verbietet ein Wahlmännersystem. Er schließt jedes Verfahren aus, bei dem sich zwischen Wähler und Wahlbewerber nach der Wahlhandlung eine Instanz schiebt, die nach ihrem Ermessen die Abgeordneten auswählt und damit den Wählern die Möglichkeit nimmt, diese selbsttätig mittels Stimmabgabe zu bestimmen. ${ }^{39}$ Die Wirkungen dieser Stimmabgabe müssen für die Wähler erkennbar sein. ${ }^{40}$

Ein Verstoß gegen den Grundsatz der Unmittelbarkeit der Wahl im Sinne des Verbots einer indirekten Wahl lag ganz offensichtlich nicht vor. Die Wirkungen der Stimmabgabe waren auch erkennbar. Das Gesetz brachte deutlich zum Ausdruck, dass der Anteil der Persönlichkeits- und Listenstimmen an den Parteistimmen für die Verteilung der auf einen Wahlvorschlag entfallenden Sitze maßgeblich ist.

36 Vgl. Bericht des Verfassungs- und Bezirksausschusses über die Prüfung der gegen die Gültigkeit der Wahlen der Bürgerschaft und zu den Bezirksversammlungen am 24. Februar 2008 eingegangenen Wahleinsprüche, Bürgerschafts-Drs. 19/627 vom 25. Juni 2008, S. 13.

37 Vgl. ebenda, S. 14.

38 Vgl. Plenarprotokoll 19/9 vom 10. Juli 2008 über die Sitzung der Bürgerschaft am 10. Juli 2008, S. 405, S. 413.

39 Vgl. Klaus David, a.a.O. (Fn. 28). Art. 6, Rn. 48 mit weiteren Nachweisen

40 Vgl. BVerfG, Beschluss vom 26. Februar 1998, in: BVerfGE 97, S. 317, S. 326; Urteil vom 10. April 1997, in: BVerfGE 95, S. 335, S. 350. 
Einwände unter Berufung auf etwaige Normenunklarheit ließen sich ebenfalls nicht begründen. Hinsichtlich der Erkennbarkeit der Wirkung, die die eigene Stimmabgabe auf den Erfolg oder Misserfolg eines Wahlbewerbers hat, gilt nichts anderes als für den Wahlrechtsgrundsatz der Unmittelbarkeit. Zur Normenklarheit ist darauf hinzuweisen, dass die Normen dem Wähler kein unzutreffendes oder unverständliches System der Wirkungsweise des Wahlrechts vorgeführt haben.

Nicht das 2007 geltende Wahlsystem als solches führte zu dem vom Beschwerdeführer beanstandeten Ergebnis, sondern das konkrete Verhalten der Wähler. Diese hatten zum Beispiel entweder mehrheitlich eine Wahlkreisliste in ihrer Gesamtheit gewählt und damit die Reihenfolge akzeptiert, in der die Bewerber darauf verzeichnet waren, oder sie hatten ihre Persönlichkeitsstimmen bevorzugt den an vorderer Stelle der Liste platzierten Kandidaten gegeben. Es liegt im Wesen der Verteilung der Mandate gemäß der Listenreihenfolge, dass Bewerber mit geringerer Zahl an Persönlichkeitsstimmen ein Mandat erwerben, andere mit einer höheren Zahl dagegen nicht. Das Hamburgische Verfassungsgericht stellte das Verfahren nach Rücknahme der Beschwerde mit Beschluss vom 7. September 2009 ein. ${ }^{41}$

\section{Erneute Volksinitiative 2008}

Das Wahlrecht des Jahres 2007 entsprach nach wie vor nicht den Vorstellungen der Initiative „Mehr Demokratie - Ein faires Wahlrecht für Hamburg“. Sie beanstandete die starren Landeslisten, die so genannten Mehrheitssicherungsklausel, das Verfahren, das an die Stelle der Relevanzschwelle getreten war, weil es nicht sicherstelle, dass sich die Persönlichkeitsstimmen allein in der Weise auswirkten, wie sie den einzelnen Kandidaten von den Wählern direkt gegeben würden, und schließlich die Fünfprozenthürde für die Wahl zu den Bezirksversammlungen.

Die Initiative bevorzugte auf der Ebene des Bürgerschaftswahlrechts stattdessen ein Wahlkreisrecht zwar nach dem geltenden System mit 17 Wahlkreisen, drei bis fünf Kandidaten und fünf Stimmen zum Kumulieren und Panaschieren, jedoch ohne Wahlkreisliste. Die Mandate sollten jene Kandidaten erlangen, die die meisten Stimmen erhalten. Die starren Landeslisten sollten wieder geöffnet werden, und es sollte keine Listenstimme mehr geben. Die Wähler sollten auch hier fünf Stimmen zum Kumulieren und Panaschieren bekommen, die allein die Listenkandidaten erhalten sollten. ${ }^{42}$

Die Initiative setzt sich dafür ein, im Bezirkswahlrecht die Fünfprozenthürde abzuschaffen und die Wahlen zu den Bezirksversammlungen am Tag der Wahl zum Europäischen Parlament abzuhalten ${ }^{43}$, um eine Dominanz von Kandidaten für die Bürgerschaft im Wahlkampf zu vermeiden. Die Wahlkreise sollten nicht identisch mit den für die Wahl zur Bürgerschaft geltenden sein, sondern lokalen Bedürfnissen angepasst werden.

Die Volksinitiative kam 2008 zustande. Das nachfolgende Volksbegehren war 2009 erfolgreich. ${ }^{44}$ Nach dem Inkrafttreten des Wahlrechts von 2009 zogen die Initiatoren aber

41 Vgl. HVerfG 04/08.

42 Vgl. Mitteilung des Senats an die Bürgerschaft, Bürgerschafts-Drs. 19/123, a.a.O. (Fn. 4).

43 Vgl. ebenda.

44 Vgl. die beiden Mitteilungen des Senats an die Bürgerschaft, a.a.O. (Fn. 4). 
ihren Antrag auf die Durchführung eines Volksentscheids zurück, der ursprünglich für den Tag der Bundestagswahl am 27. September 2009 vorgesehen war. ${ }^{45}$

\section{Das Wahlrecht von 2009}

Das 2009 geschaffene Wahlrecht besteht aus dem Zwölften Gesetz zur Änderung der Verfassung der Freien und Hansestadt Hamburg ${ }^{46}$ und dem Vierten Gesetz zur Änderung wahlrechtlicher Vorschriften. ${ }^{47}$ Beide Gesetze beruhen auf Anträgen, die interfraktionell von allen vier in der Bürgerschaft der 19. Wahlperiode vertretenen Fraktionen eingebracht wurden. ${ }^{48}$ Die Verfassungsänderung trat bewusst nach dem Vierten Änderungsgesetz in Kraft, um dessen Inhalt nicht den in Art. 1 des Verfassungsänderungsgesetzes vorgesehenen Wirkungen zu unterwerfen. Art. 2 lässt in einer Schlussbestimmung an der Nichtanwendbarkeit des Art.1 auf die bis zum Zeitpunkt seines Inkrafttretens endgültig beschlossenen Gesetze keinen Zweifel. Dem neuen Wahlrecht liegt ein Kompromiss mit „Mehr Demokratie - Ein faires Wahlrecht für Hamburg“ zugrunde, dem alle Fraktionen ausweislich der Anträge zugestimmt haben. Darin wurden - geregelt nunmehr in Art. 1 des Vierten Änderungsgesetzes - grundlegende Strukturentscheidungen übernommen, die mit dem Volksentscheid von 2004 beschlossen, in den Änderungsgesetzen von 2006 und 2007 zum Teil und im Entwurf der Volksinitiative von $2008^{49}$ beibehalten worden waren.

\subsection{Mehrmandatswahlkreise}

Gemäß $₫ 2$ Abs. 2 werden von 121 Abgeordneten mindestens 71 nach Listen in Mehrmandatswahlkreisen und die übrigen nach Landeslisten gewählt. Hamburg bleibt eingeteilt in 17 Wahlkreise gemäß der Anlage nach $₫ 18$ Abs. 8 BüWG. In ihnen werden wie bisher nach $₫ 18$ Abs. 1 Satz 1 drei bis fünf Sitze vergeben. Einzelbewerber sind weiterhin zugelassen. Wie schon 2004 und gemäß Entwurf der Initiative von 2008 sieht $\$ 25$ Abs. 1 Satz 4 vor, dass auf Landeslisten höchstens 60 Personen benannt sein dürfen.

\subsection{Zweimal fünf Stimmen}

Unterschiede weisen die Gesetze und der Entwurf bei der Zahl der zu vergebenden Stimmen auf. Das Volksgesetz von 2004, der Entwurf der Volksinitiative und das Änderungsgesetz von 2009 verleihen übereinstimmend in ihrem Art. $1 \$ 3$ Abs. 1 den Wahlberechtigten

45 Vgl. Unterrichtung durch den Präsidenten der Bürgerschaft, Bürgerschafts-Drs. 19/3648 vom 21. Juli 2009; Rücknahme des Antrags auf Durchführung eines Volksentscheids mit Schreiben der Initiatoren vom 15. Juli 2009.

46 Vom 8. Juli 2009, HmbGVBl., a.a.O. (Fn. 5), S. 221.

47 Vom 7. Juli 2009, HmbGVBl., S. 213.

48 Anträge gemäß Bürgerschafts-Drs. 19/3255 (Änderung der Verfassung) und 19/3280 (Änderung wahlrechtlicher Vorschriften), jeweils vom 10. Juni 2009.

49 Bekanntmachung des Volksbegehrens „Mehr Demokratie - Ein faires Wahlrecht für Hamburg“, in: Amtlicher Anzeiger, Nr. 97 vom 12. Dezember 2008, S. 2535, Anlage 1, S. 2541. 
fünf Stimmen für die Wahl nach Wahlkreislisten und fünf Parteistimmen - das Gesetz von 2009 spricht von Landesstimmen - für die Wahl nach Landeslisten. Die Änderungen von 2006 und 2007 beschränkten die Stimmen für die Landeslisten auf je eine, sahen im Übrigen in $\$ 3$ Abs. 1 fünf Wahlkreisstimmen vor.

Die jeweils fünf Wahlkreis- und fünf Landesstimmen erweitern seit 2009 gegenüber den Änderungsgesetzen von 2006 und 2007 die Möglichkeiten des Kumulierens und Panaschierens. Nach jenen Änderungen konnte mit der Beschränkung der Listenwahl auf eine Stimme Kumulieren und Panaschieren dort nicht mehr in Betracht kommen. Art. $1 \$ 3$ Abs. 3 des Gesetzes von 2009 stellt für die Landesstimmen diese Möglichkeiten wieder her, dies in Übereinstimmung mit dem Volkswahlgesetz von 2004, dagegen nicht ganz mit dem Entwurf der Volksinitiative von 2008. Dieser wollte ausschließen, dass Stimmen überhaupt für Landeslisten insgesamt abgegeben werden können. Im Wege des Kompromisses hat die Initiative davon Abstand genommen. $\$ 3$ Abs. 3 Nr. 3 lässt nun zu, dass Landeslisten in ihrer Gesamtheit gewählt werden können und dabei auch kumuliert und panaschiert werden kann.

Hinsichtlich der Wahlkreislisten hat sich dagegen die Initiative durchgesetzt. Wahlkreisstimmen können nicht für Wahlkreislisten in ihrer Gesamtheit gesetzt und damit auch nicht durch Kumulieren und Panaschieren vergeben werden. Durchaus abweichend von $\$$ 3 Abs. 1 Satz 2 Nr. 3 des Volkswahlgesetzes von 2004 können gemäß $₫ 3$ Abs. 2 Nrn. 1 und 2 des geltenden Rechts die Wähler ihre bis zu fünf Stimmen nur auf die in den Wahlkreislisten genannten Personen verteilen. Die Regelung über die Wirkung von Listenstimmen auf die Vergabe von Wahlkreismandaten an Bewerber gemäß $₫ 4$ Abs. 3 des Änderungsgesetzes von 2007 ist damit gegenstandslos geworden. Sie hatte ihre Existenz dem Urteil des Hamburgischen Verfassungsgerichts vom 27. April 2007 zu verdanken.

Insgesamt hat damit die Volksinitiative ihr Ziel erreicht, den Einfluss der Wähler auf die personelle Zusammensetzung der Bürgerschaft gegenüber den Änderungen von 2006 und 2007 zu stärken. ${ }^{50}$ Das Änderungsgesetz von 2007 hatte immerhin eine Hürde auf dem Weg zu diesem Ziel beseitigt, nämlich die so genannte Relevanzschwelle.

\subsection{Verhältnis der Gesamtstimmen}

Die Regeln über die Bestimmung der Gesamtzahl der Sitze in der Bürgerschaft haben sich nicht verändert. Es gelten nach Art. $1 \$ 5$ Abs. 3 und 5 in der Fassung von 2009 weiter die Hinzurechnungen und Erhöhungen zu den 121 Sitzen gemäß dem Volkswahlgesetz von 2004, der Änderung von 2006 und dem Entwurf von 2008: Überhang- und Ausgleichsmandate, Einzelbewerber, ungerade Gesamtzahl, und die so genannte Mehrheitssicherungsklausel. Letztere hat die Volksinitiative hingenommen, auch wenn sie sie als systemwidrig bezeichnet hat. ${ }^{51}$

50 Vgl. Bekanntmachung des Volksbegehrens „Mehr Demokratie - Ein faires Wahlrecht für Hamburg“, a.a.O. (Fn. 49), Allgemeine Begründung, S. 2540, Erläuterung zu Art. $1 \$ 3$ Abs. 2, S. 2541 .

51 Vgl. Bekanntmachung des Volksbegehrens „Mehr Demokratie - Ein faires Wahlrecht für Hamburg“, a.a.O. (Fn. 49), Erläuterungen zu Art. 1 \$ Abs. 4, S. 2544. 
Die Verteilung der Bürgerschaftssitze auf die Parteien und Wählervereinigungen richtet sich gemäß Art. $1 \$ 3$ Abs. 4 des Änderungsgesetzes von 2009 nach dem Verhältnis der für die Landeslisten abgegebenen Gesamtstimmen, nicht mehr nach den Parteistimmen, wie es in $\$ 3$ Abs. 2 des Volkswahlgesetzes von 2004 hieß oder einfach nach Stimmen gemäß $\$ 3$ Abs. 4 in der Fassung von 2006. Gesamtstimmen definiert $\$ 5$ Abs. 1 Nr. 4 BüWG 2009 als die Personen- und Parteistimmen, die für jede Landesliste insgesamt abgegeben wurden. Sie setzen sich also aus beiden zusammen. Personenstimmen wiederum bestehen nach der Nr. 2 des $₫ 5$ Abs. 1 aus Landesstimmen, die auf alle Personen einer Landesliste entfielen. Parteistimmen als Teil der Gesamtstimmen bezeichnet Nr. 3 des $\$ 5$ Abs. 1 als Landesstimmen, die für jede Landesliste in ihrer Gesamtheit vergeben wurden. Die 121 Sitze werden gemäß $₫ 5$ Abs. 4 nach dem Divisorverfahren mit Standardrundung auf die Landeslisten auf Grundlage der erhaltenen Gesamtstimmen verteilt. Berücksichtigt werden gemäß $₫ 5$ Abs. 2 BüWG 2009 nach wie vor nur Landeslisten, die mindestens fünf Prozent der abgegebenen gültigen Gesamtstimmen erhalten haben.

\subsection{Sitzverteilung: Verteilung der Wahlkreismandate}

Die Verteilungsregeln über die in den Wahlkreisen zu vergebenden Sitze blieben von 2004 bis 2009 in den jeweiligen Art. $1 \$ 4$ Abs. 2 der Gesetze unverändert. Jede Wahlkreisliste erhält so viele Sitze, wie sich nach Teilung der Summe ihrer Wahlkreisstimmen durch die Wahlzahl ergeben. Als Summe der Wahlkreisstimmen definiert $\$ 4$ Abs. 1 Nr. 2 die Wahlkreisstimmen, die für alle Personen einer Wahlkreisliste abgegeben wurden. Die Wahlzahl wiederum wird berechnet, indem die Zahl der insgesamt in einem Wahlkreis abgegebenen gültigen Wahlkreisstimmen durch die Zahl der im Wahlkreis zu vergebenden Sitze geteilt wird. Die danach auf einen Wahlvorschlag entfallenden Sitze weist $\$ 4$ Abs. 3 den Personen in der Reihenfolge der Stimmenzahl zu. Dies ist wie 2004 der alleinige Maßstab. Im Unterschied zu 2004 gibt es 2009 aber keine Wahlkreislistenstimmen mehr.

\subsection{Sitzverteilung: Verteilung der Listenmandate}

Gemäß Art. $1 \S 5$ Abs. 7 Satz 1 wird für jede Landesliste festgestellt, wie viele der nach Absatz 6 verbleibenden Sitze nach Listenwahl zu vergeben sind. Absatz 6 bestimmt, dass von der für jede Landesliste ermittelten Abgeordnetenzahl die Zahl der von der Partei oder Wählervereinigung in den Wahlkreisen errungenen Sitze abgerechnet wird.

Die Verteilung der Sitze an die auf einer Liste stehenden Bewerber bestimmt der Wähler sowohl mit den Stimmen, die er für die Liste insgesamt abgibt, als auch mit seinen Stimmen für bestimmte Bewerber auf der Liste. Beides ist also maßgebend für die Verteilung. Das Gesetz musste eine Methode finden, mit der auch beide Arten der Stimmvergabe für die Verteilung der Sitze an die Bewerber wirksam werden kann. Nur damit konnte es den Anforderungen des Hamburgischen Verfassungsgerichts gemäß seinem Urteil vom 27. April 2007 entsprechen.

Art. $1 \S 5$ Abs. 7 und 8 BüWG 2009 stellt einen Proporz her zwischen einerseits den Sitzen, die Personen in der Reihenfolge zugeteilt werden, in der sie auf der Landesliste stehen, und andererseits solchen Sitzen, die Personen in der Reihenfolge der Personenstim- 
menzahlen erhalten. $\$ 5$ Abs. 7 Satz 2 sieht eine Berechnungsmethode nur für die erste der beiden genannten Sitzverteilungskategorien vor. Danach wird die Zahl der Parteistimmen nach $\$ 5$ Abs. 1 Nr. 3 mit der Zahl der nach $₫ 5$ Abs. 6 verbleibenden Sitze vervielfacht und durch die Zahl der Gesamtstimmen gemäß $\$ 5$ Abs. 1 Nr. 4 geteilt. Diese Methode bewirkt, dass das Kontingent der Sitze, die in der Reihenfolge der Landesliste vergeben werden, desto größer ist, je größer die Zahl der Parteistimmen ausfällt und umgekehrt. Subtrahiert man die sich daraus ergebende Zahl von der insgesamt über eine Landesliste zu vergebenden Sitze, ergibt sich die Zahl der nach Persönlichkeitsstimmen zugeteilten Mandate. Sodann werden gemäß $₫ 5$ Abs. 8 Sätze 1 und 2 zunächst die Sitze zugeteilt, die in der Reihenfolge der Listenplätze zu vergeben sind. Anschließend erhalten die Bewerber in der Reihenfolge der Personenstimmenzahlen die restlichen Plätze. Dies entspricht dem so genannten Bremer Modell. ${ }^{52}$

Nach diesem System bestimmen die Wähler in einem ersten Schritt den Proporz zwischen der Zahl der über die Liste und der Zahl der nach Persönlichkeitsstimmen zu vergebenden Sitze. Er hängt, wie dargestellt, davon ab, wie viele Stimmen die Wähler auf eine Landesliste insgesamt und wie viele sie auf die darin aufgeführten Personen setzen. Erst damit steht fest, wie viele Kandidaten über die Liste nach Reihenfolge und gemäß Persönlichkeitsstimmen Mandate erhalten. Diese abstrakt zu nennende Proporzbestimmung geht über die konkrete Wahlentscheidung zu Gunsten einer Liste oder eines individuellen Kandidaten hinaus.

Entsprechend allgemein geübter Praxis setzen Parteien ihr politisches Spitzenpersonal auf die vordersten Plätze ihrer jeweiligen Listen. Diese Spitzenkandidaten vereinigen wegen ihres Bekanntheitsgrades im Allgemeinen die höchsten Zahlen an Persönlichkeitsstimmen auf sich. Dementsprechend wirkt sich ein solches Wahlverhalten auf den Proporz zu Gunsten der Zahl der Sitze aus, die nach Persönlichkeitsstimmen zu vergeben sind. Da zuerst aber die Mandate an die Listenbewerber gemäß Reihenfolge vergeben werden, kann der Fall eintreten, dass zum Beispiel der an erster Stelle der Liste stehende Spitzenkandidat zwar die höchste Zahl an Persönlichkeitsstimmen auf sich vereinigt, gleichwohl aber sein Mandat über die Liste erhält, ungeachtet der Frage, ob er in einem Wahlkreis ein Direktmandat erworben hat. In jedem Fall nimmt dieser Spitzenkandidat nicht das Kontingent der Sitze in Anspruch, die nach den Persönlichkeitsstimmen vergeben werden. Als weitere Folge kann eintreten, dass auf diesem Kontingent ein Kandidat nachrückt, nämlich der mit der nächstniedrigen Zahl an Persönlichkeitsstimmen. Im Ergebnis also erhält ein Bewerber ein Mandat mit einer deutlich niedrigeren Stimmenzahl, als sie der Spitzenkandidat erzielt hat, obwohl die Wähler an ihn ausweislich seiner vergleichsweise geringen Stimmenzahl kaum gedacht haben dürften.

52 Vgl. $\$ 7$ Abs. 6 des Bremischen Wahlgesetzes vom 23. Mai 1990, Brem.GBl. S. 321, zuletzt geändert am 19. Dezember 2006, Brem.GBl. S. 539. Vgl. auch das Gesetz zur Änderung des Bremischen Wahlgesetzes auf Grund des Vorschlags der Initiative „Mehr Demokratie“- Drs. 16/863 vom 12. Dezember 2005, B. Besonderer Teil: Zu den einzelnen Vorschriften, zu Nummer 2 (\$ 7). Der Staatsgerichtshof der Freien Hansestadt Bremen hat $\$ 7$ Abs. 6 mit Urteil vom 8. April 2010 (St 3/09) für verfassungsgemäß erklärt. 


\subsection{Wahlen zu den Bezirksversammlungen}

Das Recht der Wahlen zu den Bezirksversammlungen regeln die Artikel 2 und 3 des Vierten Änderungsgesetzes neu. Die Verweisungsnorm des Art. $2 \$ 1$ Abs. 1 bleibt erhalten. Danach finden auf die Wahlen zu den Bezirksversammlungen die Vorschriften des Gesetzes über die Wahl zur hamburgischen Bürgerschaft entsprechende Anwendung. Wie bereits das Volkswahlgesetz von 2004 und der Entwurf von 2008 bestimmt das Bezirkswahlrecht von 2009 in seinem Art. $2 \$ 2$ Abs. 1, dass die Wahl zu den Bezirksversammlungen am Tag der Wahl zum Europäischen Parlament stattfindet.

Geben und Nehmen kennzeichnet auch das neue Bezirkswahlrecht. Hatte die Volksinitiative die vorgenannten Positionen durchgesetzt, so hat sie im Unterschied zum Volkswahlgesetz von 2004 und ihrem Entwurf von 2008 hingenommen, dass bei der Sitzverteilung nur Bezirkslisten berücksichtigt werden, die mindestens drei Prozent der insgesamt gültigen Stimmen erhalten haben. Die Bürgerschaft hat somit gegenüber dem Wahlrecht des Jahres 2006 immerhin zwei Prozentpunkte von fünf auf drei hergegeben.

Gelöst vom bisher geltenden Recht hat sich das Bezirkswahlrecht auch hinsichtlich der Wahlkreise. Entsprach deren Einteilung bisher derjenigen für die Bürgerschaftswahl, so soll jetzt örtlichen Bedürfnissen Rechnung getragen werden. Gemäß Art. $2 \$ 3$ Abs. 1 Satz 1 beschließt die Bezirksversammlung wie schon nach dem Volkswahlgesetz von 2004 über die Wahlkreiseinteilung. Verfassungsrechtlichen Bedenken gegen diese damals allein den Bezirksversammlungen verliehene Befugnis begegnet das Gesetz dadurch, dass die Wahlkreiseinteilung wie im Bürgerschaftswahlrecht als Anlage zum Gesetz von der Bürgerschaft bestimmt wird. ${ }^{53}$ Damit erhält das Recht der Bezirksversammlungen den Charakter eines zumindest politisch bindenden Vorschlags.

\section{Die Verfassungsänderung}

Das Zwölfte Gesetz zur Änderung der Verfassung der Freien und Hansestadt Hamburg beruht ebenso wie das Vierte Änderungsgesetz auf einer Verständigung der in der Bürgerschaft vertretenen Parteien mit der Volksinitiative „Mehr Demokratie - Ein faires Wahlrecht für Hamburg “. ${ }^{54}$ Die für die Wahl zur Bürgerschaft in den Sätzen 2 bis 4 des Art. 6 Abs. $4 \mathrm{HV}$ eingeführten Änderungen gelten nach einem in Art. 4 Abs. $2 \mathrm{HV}$ angefügten Satz auch für die Wahl der Bezirksversammlungen.

Das Ziel der Verfassungsänderung besteht im Wesentlichen darin, sowohl Beschlüsse der Bürgerschaft als auch Entscheide des Volkes über Wahlgesetze qualifizierten Anforderungen auszusetzen. Zunächst gewinnen diese Qualifizierungen materiell an Bedeutung. Gemäß dem neu gefassten Satz 1 des Art. 6 Abs. 4 HV bestimmt das Gesetz das Nähere. Bisher

53 Vgl. Bekanntmachung des Volksbegehrens „Mehr Demokratie - Ein faires Wahlrecht für Hamburg“, a.a.O. (Fn. 49), Erläuterung zu Art. 2, \$3 Abs. 1 und 2, S. 2567. Die Bedenken beruhten darauf, dass die Bezirksversammlungen keine Gesetzgebungsbefugnis besitzen, die Wahlkreiseinteilung gesetzlich aber zu regeln ist.

54 So ausdrücklich die Begründung zum Antrag gemäß Bürgerschafts-Drs. 19/3255, a.a.O. (Fn. 48), I. Allgemeines, S. 2. 
galt, dass dieses nur das Nähere über die Zahl der Abgeordneten, das Wahlrecht, die Wählbarkeit und die Durchführung der Wahl festlegen sollte. Der neue Satz 1 entspricht Art. 38 Abs. 3 GG und übernimmt damit dessen Regelungsbereich für das hamburgische Wahlrecht. Gesetze im Sinne des Satzes 1 und damit auch des Satzes 3 sind nur die einfachen, soweit die Bürgerschaft sie beschließt. Für die Änderung des in der Verfassung verankerten Wahlrechts durch die Bürgerschaft gilt nach wie vor Art. $51 \mathrm{HV}$.

\subsection{Zukünftige Änderungen im Konsens}

Erklärtes Ziel der Änderung ist unter anderem zu gewährleisten, dass Wahlrechtsänderungen seitens der Bürgerschaft nur in einem weitgehenden Konsens der in ihr vertretenen Fraktionen beschlossen werden. Derartige Änderungen bedürfen künftig einer qualifizierten Mehrheit von zwei Dritteln der Abstimmenden. Auf ein Mindestquorum wie in Art. 51 Abs. 2 für Verfassungsänderungen wurde bewusst verzichtet. ${ }^{55}$

Damit erweist sich das Quorum als stumpf im Hinblick auf das erklärte Ziel. Zunächst bildet nur die Zahl der abgegebenen Stimmen, nicht etwa die der anwesenden Abgeordneten die Grundlage. Die Begründung spricht ausdrücklich allein von „an der Abstimmung teilnehmenden Abgeordneten" "56. Sodann lässt der reine Wortlaut das Verständnis zu, dass auch sich als ungültig erweisende Stimmen zu den abgegebenen zählen. Schließlich setzt die Feststellung der Ungültigkeit die Abgabe voraus. Auf diese denkbare Bedeutung war bereits das Hamburgische Verfassungsgericht gestoßen. Es hatte allerdings nicht zu entscheiden, ob mit den abgegebenen nur die abgegebenen gültigen Stimmen gemeint sein sollten. ${ }^{57}$

Die Verfassungsänderung verzichtet auf jedes Quorum, auch auf eine Mindestzahl der Abstimmenden. Es würde theoretisch für einen wirksamen Gesetzesbeschluss ausreichen, wenn drei Stimmen abgegeben werden, von denen zwei dem Gesetzentwurf zustimmen. Die Beschlussfähigkeitsregelung des Art. $20 \mathrm{HV}$ verhindert dies nicht. Die Beschlussfähigkeit besteht bei Anwesenheit von mehr als der Hälfte der Mitglieder der Bürgerschaft. Die Anwesenden bilden weder ein Quorum, wie sich gezeigt hat, noch müssen sie an der Abstimmung teilnehmen. Selbst wenn bei insgesamt 121 Mitgliedern 61 anwesend sind und diese an der Abstimmung teilnehmen, reicht für die Zweidrittelmehrheit eine Zustimmung von 41 Stimmen aus. Diese Zahl zu erreichen bedarf keines „weitgehenden Konsenses in der Bürgerschaft" für Wahlrechtsänderungen, zumal diese Mehrheit in Anbetracht der mangelnden Stringenz der Bestimmung durchaus mit weniger Stimmen zu erreichen ist, zum Beispiel bei einer geringeren Zahl der Abstimmenden trotz bestehender Beschlussfähigkeit. Die erforderliche Zahl an Stimmen kann unter Umständen eine Fraktion allein aufbringen. Die politisch-parlamentarische Wirklichkeit wird wohl anders aussehen.

55 Begründung zum Antrag gemäß Bürgerschafts-Drs. 19/3255, a.a.O. (Fn. 48), II. Einzelbegründung zu Art. 1 Nr. 2, S. 3.

56 Vgl. ebenda.

57 Vgl. Urteil, a.a.O. (Fn. 24), S. 76. 


\subsection{Verlangen der Wahlberechtigten}

Der neue Satz 3 des Art. 6 Abs. 4 HV erklärt Art. 50 Abs. 4 Sätze 1 bis 4 HV in der Fassung des Elften Gesetzes zur Änderung der Verfassung der Freien und Hansestadt Hamburg $^{58}$ für anwendbar. Gemäß Art. 50 Abs. 4 Satz 1 HV tritt ein von der Bürgerschaft beschlossenes Gesetz, das ein vom Volk beschlossenes Gesetz aufhebt oder ändert, nicht vor Ablauf von drei Monaten nach seiner Verkündung in Kraft. Diese Fassung entspricht einem Vorschlag der Volksinitiative. Damit soll die Verbindlichkeit von Volksentscheiden wesentlich erhöht werden. ${ }^{59}$ Innerhalb dieser Frist können 2,5 Prozent der Wahlberechtigten einen Volksentscheid über das bürgerschaftliche Änderungsgesetz verlangen. Geschieht dies, tritt das Änderungsgesetz nicht vor Durchführung des Volksentscheids in Kraft. All dies galt schon seit Ende 2008 auch für bürgerschaftliche Wahlgesetze, die Volkswahlgesetze ändern. Die Besonderheit des Art. 6 Abs. 4 Satz 3 besteht darin, dass die Dreimonatsfrist und das Verlangensrecht mit den weiteren Folgen sich auf dem Gebiet des Wahlrechts nicht auf bürgerschaftliche Gesetze beschränken, die Volkswahlgesetze ändern. Vielmehr bestehen die genannten Regelungen unabhängig davon für jedes Wahlgesetz der Bürgerschaft ${ }^{60}$, sei die Änderung noch so geringfügig. Gegenstand des Volksentscheids ist in jedem Fall das bürgerschaftliche Änderungsgesetz, nicht etwa ein Entwurf einer Volksinitiative.

\subsection{Volksentscheide}

Der Volksentscheid über ein bürgerschaftliches Wahlgesetz findet, wenn mindestens 2,5 Prozent der Wahlberechtigten ihn fristgerecht verlangt haben, grundsätzlich am Tag der Wahl zur Bürgerschaft oder zum Bundestag statt (Art. 50 Abs. 3 Satz 7 HV). Dies schiebt das Inkrafttreten des Gesetzes in noch weitere Ferne. Die Bürgerschaft kann aber verlangen, dass der Volksentscheid auch an einem anderen Tag stattfindet, was im Hinblick auf die Seltenheit von Bürgerschafts- und Bundestagswahlen als mehr als sinnvoll erscheint. Das Parlament muss also die Initiative ergreifen. Für diesen Fall sieht Satz 3 des Art. 6 Abs. 4 HV für das Zustandekommen des Volksentscheids eine neue qualifizierte Mehrheit vor. Das Gesetz bedarf einer Mehrheit von zwei Dritteln der Abstimmenden und der Zustimmung von einem Fünftel der Wahlberechtigten.

Liegt die Initiative zur Wahlgesetzgebung beim Volksbegehren, gilt Art. 50 Abs. 3 Satz $11 \mathrm{HV}$ entsprechend. Es gelten die Mehrheiten für Verfassungsänderungen als Gegenstand eines Volksentscheids, nämlich eine Mehrheit von zwei Dritteln der Abstimmenden und von mindestens zwei Dritteln der in dem gleichzeitig gewählten Parlament repräsentierten Hamburger Stimmen. Wie damit umzugehen ist, erklärt Satz 12. Die Volksinitiative kann nicht beantragen, dass ein von ihr auf dem Gebiet des Wahlrechts initiierter Volksentscheid an einem anderen Tag als dem einer Bürgerschafts- oder Bundestagswahl stattfindet. Ande-

58 Vom 16. Dezember 2008, HmbGVBl. S. 431.

$59 \mathrm{Vgl}$. Antrag, betreffend 11. Gesetz zur Änderung der Verfassung der Freien und Hansestadt Hamburg, Bürgerschafts-Drs. 19/1476 vom 5. November 2008, Begründung II. Zu Absatz 4, S. 5.

60 Vgl. Bekanntmachung des Volksbegehrens „Mehr Demokratie - Ein faires Wahlrecht für Hamburg", a.a.O. (Fn. 48), I. Allgemeines, S. 2. 
renfalls liefe die Quorumsregelung leer. Die Verfassungsänderung hat bemerkenswert viele und unterschiedliche Mehrheitsregeln mit sich gebracht, noch dazu in einer einzigen Norm.

\section{Erkenntnisse aus dem Verlauf der Hamburger Wahlrechtsänderungen}

\subsection{Erfolg der Volksinitiative}

Am Anfang stand 2004 ein erfolgreicher Volksentscheid. Dem daraus hervorgegangenen Recht blieb die umfassende Anwendung in einer Wahl versagt. Am Ende steht 2009 ein diesem Recht im Wesentlichen entsprechendes Gesetz, das als Grundlage der nächsten Wahl dienen wird, ohne dass es mit einem Volksentscheid beschlossen wurde. Für die Volksinitiative „Mehr Demokratie - Ein faires Wahlrecht für Hamburg“ liegt darin jedenfalls insofern ein Erfolg, als sie mit der Durchsetzung des von ihr geforderten materiellen Wahlrechts im Wesentlichen das Ziel ihrer Wünsche erreicht hat. Der Weg dahin verlief aus ihrer Sicht allerdings nicht ohne negative Erfahrungen im Umgang mit Volksentscheiden. Dazu gehört nicht nur die Änderung des Volkswahlgesetzes von 2004 durch die Novelle von 2006, sondern auch die Nichtberücksichtigung des Volksentscheides gegen den teilweisen Verkauf des Hamburger Landesbetriebes Krankenhäuser 2004. Letzterer hatte zwar nichts mit Wahlrecht zu tun. Wohl aber haben beide Ereignisse auf Seiten der Volksinitiative das Bedürfnis nach einer Verbindlichkeit von Volksentscheiden gesteigert. Die Initiative „Hamburg stärkt den Volksentscheid“ scheiterte 2007 allerdings daran, mittels Volksentscheids die Verbindlichkeit in der Verfassung zu verankern. Erfolg hatten die Bemühungen darum mit der Verfassungsänderung des Jahres 2009 speziell für das Wahlrecht, im Übrigen bereits Ende 2008 für andere Materien durch entsprechende Änderung des Art. 50 HV.

8.2. Die Bürgerschaft auf dem Weg zum Kompromiss von 2009 - Preisgegebene Grundsätze

Die einzelnen Schritte, die die Bürgerschaft auf dem Weg zur grundlegenden Systemveränderung des Wahlrechts seit 2004 gegangen ist, haben ihr jeweils angepasste, veränderte Begründungen abverlangt, mit denen sie sich von zuvor geltenden Grundsätzen verabschiedet hat.

Eine der grundlegenden Systemveränderungen liegt in der Einführung von Wahlkreisen, in denen Direktkandidaten gewählt werden. Mit dieser Frage hatte sich die Bürgerschaft schon 1992 bei der parlamentarischen Behandlung des Berichts der Enquete-Kommission „Parlamentsreform“ zu befassen. Die Kommission hatte die Herabsetzung der Zahl der Abgeordneten auf 101 und die Einführung eines personalisierten Verhältniswahlrechts mit 41 in Wahlkreisen und 60 über Landeslisten zu wählenden Abgeordneten vorgeschlagen. ${ }^{61}$ Die Bürgerschaft beließ es jedoch beim System der gebundenen Listen mit einer Stimme.

61 Vgl. Bürgerschafts-Drs., a.a.O. (Fn. 8), S. 32 ff., 40 ff.; Bericht, a.a.O. (Fn. 8), S. 40 f., S. 48 ff., S. $272 \mathrm{f}$. 
Deutlich grundsätzlichere Positionen ergaben sich im Zusammenhang mit der Verfassungsreform des Jahres 1996. Teil der Reform war die Verabschiedung eines neuen Art. 13 Abs. 2 Satz 1 HV: „Die Vereinbarkeit des Amtes einer oder eines Abgeordneten mit einer Berufstätigkeit ist gewährleistet." Den damit beschriebenen Charakter der Bürgerschaft als Teilzeitparlament wollte die Fraktion der CDU nur gesichert sehen, wenn das damals in der Diskussion befindliche Wahlrecht keine Wahlkreise vorsähe. In einer dann notwendig werdenden Arbeit in den Wahlkreisen erblickte sie einen mit einer Berufstätigkeit nicht mehr zu vereinbarenden Aufwand und eine Entwicklung in Richtung auf ein Berufspolitikertum. Sie sah in Wahlkreisen das Teilzeitparlament und damit einen tragenden Pfeiler der gesamten Verfassungsreform bedroht. Die Wahlrechtsreform scheiterte. ${ }^{62}$

Von diesem Problem war acht Jahre später nicht mehr die Rede, trotz unveränderten Fortbestandes des Art. 13 Abs. 2 Satz 1 HV. Die Bürgerschaft fand sich 2004 zu einem ersten Schritt in Richtung Systemveränderung bereit. Der Entwurf, den sie als Alternative zur Vorlage der Volksinitiative zur Entscheidung stellte, sah 50 Wahlkreise vor, in denen je ein Kandidat zu wählen war. In der Stellungnahme der Bürgerschaft war durchaus die Rede von Arbeit in den Wahlkreisen, aber nicht mit Blick auf die 1996 vermeintlich bestehenden Bedrohungen. Vielmehr sei die Arbeit der Bewerber um Mandate in 50 kleinen Wahlkreisen bürgernäher zu leisten als in 17 großen. Konkrete Erfahrungen in der Zeit zuvor können diesen Sinneswandel nicht herbeigeführt haben, denn Wahlkreise gab es bis 2004 nicht. Plausibler erscheint die Annahme, dass die Bürgerschaft, wenn sie sich schon zu einem Alternativentwurf entschloss, nicht mit der Aufrechterhaltung des alten, sondern mit einer wie auch immer ausgestalteten Variante eines Wahlkreissystems aufwarten musste.

Bei den Novellen der Jahre 2006 und 2007 spielten Risiken für das Teilzeitparlament nicht mehr die mindeste Rolle. Die Bürgerschaft kam dem Volkswahlgesetz mit der Übernahme des Systems der 17 Wahlkreise, in denen 71 Kandidaten zu wählen waren, entgegen. Die noch zwei Jahre zuvor ins Feld geführte Begründung für 50 kleinere Wahlkreise, nämlich bürgernähere Arbeit der Bewerber, konnte ebenfalls nicht mehr gelten. Vielmehr kam es darauf an, durch Rückkehr zum System der gebundenen Landeslisten mit nur einer Listenstimme statt fünf pro Wähler den Einfluss der Parteien auf die Zusammensetzung des Parlaments zu sichern und vermeintliche Fehlermöglichkeiten beim Wahlvorgang zu reduzieren.

Der Kompromiss des Jahres 2009 entzog diesen Argumenten die Tragfähigkeit. Mit ihnen sollte ein Listenwahlrecht mit fünf Stimmen sowie Kumulieren und Panaschieren verhindert werden, das nun aber doch gilt. Als Probe aufs Exempel, dass Wähler mit fünf Stimmen, mit Kumulieren und Panaschieren jedenfalls in den Wahlkreisen weitgehend fehlerfrei umgehen können, mag die Zahl von gerade acht Einsprüchen gegen die Wahl vom 24. Februar 2008 dienen. Auf eine Fehleranfälligkeit des Wahlsystems rekurrierte keiner. ${ }^{63}$ Eine etwas höhere Zahl ungültiger Stimmen in den Wahlkreisen sollte in Anbetracht dessen, dass es sich um die erstmalige Anwendung des neuen Wahlrechts handelt, nicht überbewertet werden. Das Argument der Fehlerreduzierung entbehrt jedenfalls noch einer em-

62 Vgl. Klaus David, a.a.O. (Fn. 27), Vorbemerkungen, Rn. 31 und ders., Die Rechtsstellung der Bürgerschaftsabgeordneten nach der Verfassungsreform von 1996, in: Jan Albers / Klaus Asche / Jürgen Gündisch / Hans-Joachim Seeler / Werner Thieme (Hrsg.), Recht und Juristen in Hamburg, Bd. II, Köln 1999, S. 45.

63 Vgl. Bericht des Verfassungs- und Bezirksausschusses, a.a.O. (Fn. 36). 
pirisch zu belegenden Überzeugungskraft. Dies kann wohl auch für ein Wahlrecht mit zehn Stimmen angenommen werden.

\subsection{Konsens}

Die Erkenntnis, dass Konsens in auch einfach-gesetzlich zu regelndem Wahlrecht eine Streit vermeidende Wirkung entfaltet, gilt traditionell innerparlamentarisch zwischen den in einem Parlament vertretenen Fraktionen. Auf Einvernehmen hinzuwirken, berücksichtigt zudem die Bedeutung des Wahlrechts als alle Parteien betreffenden Staatsrechts. Bemerkenswert an der Wahlrechtsnovelle von 2009 ist, dass Konsensbildung die Begrenzung auf den innerparlamentarischen Raum verlassen hat. Konsens zwischen Parlament und Volk bildete jedenfalls im Ergebnis die Grundlage der Reform, nicht Wettbewerb zwischen beiden. Dieser Einsicht beugten sich in diesem Fall alle Beteiligten. Gerade die Hartnäckigkeit der Auseinandersetzungen in den vergangenen Jahren mag diesem Gewinn an Einsicht dienlich gewesen sein. Zweifel an der Haltbarkeit dieser Einsicht und des Konsenses für die Zukunft scheinen jedenfalls nicht ausgeräumt zu sein. Das belegt das Bedürfnis nach Sicherung gegen eine Aufkündigung des Konsenses mittels Regeln über Verbindlichkeit.

\subsection{Sicherung von Verbindlichkeit und Autonomie der Bürgerschaft}

Nach den Erfahrungen mit dem Volkswahlgesetz von 2004, novelliert von der Bürgerschaft 2006, und der Nichtbeachtung des Volksentscheides gegen den Verkauf des Landesbetriebes Krankenhäuser musste den Volksinitiatoren daran gelegen sein, einen größeren, möglichst hohen Grad an Verbindlichkeit von Volksentscheiden zu sichern. Dies gelang mit Verfassungsrang. Das Elfte Gesetz zur Änderung der Verfassung der Freien und Hansestadt Hamburg novellierte Art. 50 in diesem Sinn, und zwar ebenfalls im Konsens aller Beteiligten. Dies entsprach der Absicht der Koalitionsparteien der 19. Wahlperiode der Bürgerschaft. Sie hatten angestrebt, mit der Volksinitiative und den Fraktionen Gespräche über Regelungen für die Verbindlichkeit von Volksentscheiden und über die Änderung von $\mathrm{Zu}$ stimmungsquoren zu führen. ${ }^{64}$

Die Regeln über die Verbindlichkeit von Wahlrecht in den Artikeln 6 und 4 HV gehen einen bedeutenden Schritt darüber hinaus. Gegenstand des Volksentscheides, den 2,5 Prozent der Wahlberechtigten gemäß dem anwendbaren Art. 50 Abs. 4 Satz 2 verlangen können, bildet in jedem Fall ein Gesetz der Bürgerschaft. Außerhalb des Anwendungsbereichs der Art. 6 und $4 \mathrm{HV}$ hat dieses Gesetz ein vom Volk beschlossenes Gesetz geändert oder aufgehoben. Das Verlangensrecht hat also vorher gesetztes Volksrecht zur Grundlage und sichert dies. Der Volksentscheid legitimiert die bürgerschaftlichen Änderungen daran ${ }^{65}$ oder weist sie zurück. All dies trifft im Rahmen der Art. 6 und $4 \mathrm{HV}$ auf bürgerschaftlich beschlossene Wahlgesetze nicht zu. Das Verlangensrecht und ein gegebenenfalls folgender Volksentscheid dienen nicht der Sicherung vorangegangenen, von der Bürgerschaft etwa geänderten, Volkswahlrechts. Vorangegangene Änderungen hat es gar nicht gegeben. Viel-

64 Vgl. Antrag, a.a.O. (Fn. 59), Begründung: I. Allgemeines, S. 2 f.

65 Vgl. ebenda, Begründung: II. Zu Absatz 4, S. 5. 
mehr dienen die Vorschriften der Erschwerung der Änderung von Wahlrecht auf eigene Initiative der Bürgerschaft.

Diese kann also nicht sicher sein, dass von ihr beschlossenes Wahlrecht, das Volkswahlrecht nicht aufhebt oder ändert, tatsächlich Geltung erlangt. Zunächst tritt das Gesetz ohnehin nicht vor Ablauf von drei Monaten nach seiner Verkündung in Kraft. Die Bürgerschaft muss abwarten, ob innerhalb dieser Frist das genannte Verlangen zustande kommt. In diesem Fall räumt der anwendbare Art. 50 Abs. 4 Satz 4 HV dem Volk die Möglichkeit der letztverbindlichen Entscheidung über das Änderungsgesetz der Bürgerschaft ein, nicht der Bürgerschaft selbst. Bis zur Durchführung des Volksentscheides, sollte es zu ihm kommen, verlängert sich die Periode des Nichtinkrafttretens nach Art. 50 Abs. 4 Satz 3 HV über die drei Monate nach Satz 1 hinaus. Bestätigt der Volksentscheid das bürgerschaftliche Gesetz, liegt darin formal die Aufhebung der Bedingung für das Inkrafttreten, materiell die Legitimierung des Gesetzes. Mit dem zustimmenden Volksentscheid ergeht kein volksbeschlossenes Gesetz. Sollte die Bürgerschaft das letztlich vom Volk bestätigte Gesetz ändern wollen, wiche sie nicht von einem Volksgesetz ab. Art. 50 Abs. 4 HV wäre daher nicht direkt anwendbar, wohl aber mittels Art. 6 Abs. 4 Satz 3 und Art. 4 Abs. 2 Satz 3.

Damit ist die Frage aufgeworfen, ob diese über die eingangs genannte Sicherung hinausgehenden Bindungswirkungen, denen die Wahlgesetzgebung der Bürgerschaft ohne vorangegangenes Volkswahlgesetz unterliegt, nicht die Autonomie des Verfassungsorgans Bürgerschaft über Gebühr berühren. Schließlich hängt der gesetzgeberische Erfolg des Parlaments von Entscheidungen der Volksinitiatoren, gegebenenfalls vom Staatsorgan Volk ab. Damit tritt das Volk an die Seite der Bürgerschaft. Die Verfassung begründet insoweit eine Mitverantwortung des Volkes für parlamentarische Gesetzgebung. Zwei Organe handeln in diesem Fall zusammen. Nach der Konzeption der Art. 48 und 50 HV aber fassen sie ihre jeweiligen Beschlüsse regelmäßig autonom, im Fall des Art. 50 Abs. 3 Satz 6 als Konkurrenten, wenn die Bürgerschaft einen eigenen Gesetzentwurf beifügt. Diese Fragen bedürfen gesonderter Prüfung.

\subsection{Das Mittel eines Kompromisses - Spekulation über Gründe}

Wie dargelegt ist das von der Bürgerschaft 2009 beschlossene Wahlrecht zwar nicht vollständig identisch mit dem Volkswahlrecht des Jahres 2004 und dem Entwurf der Volksinitiatoren von 2008, stimmt aber bis in Einzelheiten mit beiden überein. In Anbetracht dessen hätte es zwei andere Möglichkeiten gegeben, die im Wesentlichen zu demselben Ziel geführt hätten. Die Bürgerschaft hätte zum einen gemäß Art. 50 Abs. 3 Satz 3 HV das vom Volksbegehren eingebrachte Gesetz verabschieden können. Dies ist nicht geschehen. Das Gesetz hätte zum anderen, wie ursprünglich auch beabsichtigt, am 27. September 2009 zum Gegenstand eines Volksentscheids gemacht werden können, der berechtigte Aussichten auf Erfolg gehabt hätte.

Wäre der eine oder der andere Weg beschritten worden, hätte einerseits die Volksinitiative nicht die Reform der Art. 6 und 4 HV erreicht, die Bürgerschaft andererseits die Mehrheitssicherungsregel und die Möglichkeit der Stimmabgabe für Landeslisten in ihrer Gesamtheit für die Bürgerschaftswahl sowie die Dreiprozenthürde für die Wahlen zu den Bezirksversammlungen hergeben müssen, um nur das Wesentliche zu erwähnen. Insbesondere an der Möglichkeit der Stimmabgabe für Landeslisten insgesamt musste Parteien gele- 
gen sein, die ein Interesse daran haben, bestimmten Kandidaten durch entsprechende Platzierung auf der Liste zu einem Mandat zu verhelfen. Dies kann gelingen, wenn diese Parteien von der Erwartung ausgehen, dass ihre Wähler bevorzugt Listen an Stelle von Personen wählen. In der Möglichkeit des Panaschierens und Kumulierens liegt dafür immerhin ein Risiko.

Bei jedem der beiden genannten Wege hätte sich die Bürgerschaft aber die Frage gefallen lassen müssen, warum 2009 ein von der Volksinitiative entworfenes Wahlrecht, sei es von ihr übernommen, sei es durch Volksentscheid beschlossen, möglich sein sollte, das nur drei Jahre zuvor nicht, jedenfalls nicht vollends hatte gelten sollen, wie 2006 die Novelle des Volkswahlgesetzes von 2004 gezeigt hatte. Bürgerschaftliche Wahlgesetzgebung zwischen 2006 und 2009 hätte wohl als eine zeitlich begrenzte Zwischenphase angesehen werden können. Daran hätte auch eine vollständige, aber eben nur rezeptive und damit nicht durch inhaltliche Gestaltung zu beeinflussende Übernahme des Entwurfs der Volksinitiatoren von 2008 über Art. 50 Abs. 3 Satz 3 HV nichts geändert. Darüber hinaus wäre jeder dieser beiden Fälle einem Bedeutungsverlust parlamentarischer Gesetzgebung gleichgekommen, an dem die Bürgerschaft kein Interesse haben konnte. Unter dieser Perspektive hätte sie einen erfolgreichen Volksentscheid 2009 wohl als Nachteil empfinden können, den es zu vermeiden galt. Sie musste aus ihrem Selbstverständnis heraus als mit gestaltender Teilnehmer im Spiel bleiben. Diese Rolle konnte sie nur noch im Wege der Aushandlung des Kompromisses und mit dessen gesetzgeberischer Umsetzung spielen.

An einem Kompromiss mussten die Volksinitiatoren ebenfalls interessiert sein, wenn auch aus anderen Gründen. Hätte die Bürgerschaft den Gesetzentwurf der Initiatoren unverändert übernommen, hätte sie ein parlamentarisches Wahlgesetz geschaffen. Dessen nachträgliche Änderung hätte nicht die Wirkungen des Art. 50 Abs. 4 HV ausgelöst, weil darin keine Änderung eines vom Volk beschlossenen Gesetzes gelegen hätte. Den Initiatoren musste daran gelegen sein, Sicherheiten zu erhalten, um diese aus ihrer Sicht missliebigen Folgen zu verhindern. Dies geschah mit dem neuen Art. 6 Abs. 4 und Art. 4 Abs. 2 Satz 3 HV. Es konnte nur mit einem Kompromiss gelingen.

\subsection{Folgenreiche Unterlassungen?}

Abschließend darf über die Vorstellung spekuliert werden, welche Entwicklung das Wahlrecht genommen hätte, wäre die Bürgerschaft 1992 etwa den Vorschlägen der EnqueteKommission „Parlamentsreform“ gefolgt oder hätte sich im Zusammenhang mit der Verfassungsreform von 1996 zu einer Wahlrechtsreform entschlossen, verbunden jeweils mit der Einführung von Wahlkreisen. Darin hätten wohl strategische Weichenstellungen liegen können mit Wirkungen schon auf Initiativen oder aber auf einen Erfolg einer Volksgesetzgebung auf dem Gebiet des Wahlrechts. Jene Unterlassungen dürften, von heute aus gesehen, die Bemühungen der Volksinitiatoren um Volksgesetzgebung zum Wahlrecht, insbesondere hinsichtlich der Einführung von Wahlkreisen, wohl begünstigt haben. Insofern muss sich die Hamburgische Bürgerschaft Defizite in der Wahrnehmung ihrer Repräsentativfunktion vorhalten lassen 